\title{
The TOP-SCOPE Survey of PGCCs : PMO and SCUBA-2 \\ Observations of 64 PGCCs in the Second Galactic Quadrant
}

\section{Zhang, Chuan-Peng}

2018-06

Zhang , C-P , Liu , T , Yuan , J , Sanhueza , P , Traficante , A , Li , G-X , Li , D , Tatematsu , K, Wang , K, Lee , C W , Samal , M R, Eden, D, Marston , A , Liu , X-L , Zhou , J-J , Li , P S , Koch , P M , Xu , J-L , Wu , Y, Juvela , M , Zhang , T , Alina, D , Goldsmith , P F , Toth , L , Wang , J-J \& Kim , K-T 2018 , ' The TOP-SCOPE Survey of PGCCs : PMO and SCUBA-2 Observations of 64 PGCCs in the Second Galactic Quadrant ' , Astrophysical Journal Supplement Series , vol. 236 , no. 2 , 49 . https://doi.org/10.3847/1538-4365/aac513

http://hdl.handle.net/10138/308377

https://doi.org/10.3847/1538-4365/aac513

cc_by_nc_sa

publishedVersion

Downloaded from Helda, University of Helsinki institutional repository.

This is an electronic reprint of the original article.

This reprint may differ from the original in pagination and typographic detail.

Please cite the original version. 


\title{
The TOP-SCOPE Survey of PGCCs: PMO and SCUBA-2 Observations of 64 PGCCs in the Second Galactic Quadrant
}

\author{
Chuan-Peng Zhang ${ }^{1,2,3}$ (D) Tie Liu ${ }^{4,5}$ (D) , Jinghua Yuan ${ }^{1}$ (D), Patricio Sanhueza ${ }^{6}$ (D), Alessio Traficante ${ }^{7}$, Guang-Xing Li $^{8}$, Di Li $^{1,3,9}$ (D) $^{\text {, }}$ \\ Ken'ichi Tatematsu $^{6}$ (D), Ke Wang ${ }^{10}$ (D), Chang Won Lee ${ }^{4,11}$, Manash R. Samal ${ }^{12}$, David Eden ${ }^{13}$, Anthony Marston ${ }^{14}$ (D), \\ Xiao-Lan Liu ${ }^{1}$, Jian-Jun Zhou ${ }^{15}$, Pak Shing $\mathrm{Li}^{16}{ }^{\text {(D) }}$, Patrick M. Koch ${ }^{17}$ (D), Jin-Long Xu ${ }^{1}$, Yuefang Wu ${ }^{18}$ (D), Mika Juvela ${ }^{19}$, \\ Tianwei Zhang $^{18}$ (D), Dana Alina ${ }^{20}$, Paul F. Goldsmith ${ }^{21}$ (D) L. V. Tóth ${ }^{22,23}$ (D), Jun-Jie Wang ${ }^{1}$, and Kee-Tae Kim ${ }^{4}$ (iD \\ ${ }^{1}$ National Astronomical Observatories, Chinese Academy of Sciences, 100012 Beijing, People's Republic of China; cpzhang@nao.cas.cn \\ ${ }^{2}$ Max-Planck-Institut für Astronomie, Königstuhl 17, D-69117 Heidelberg, Germany \\ ${ }^{3}$ CAS Key Laboratory of FAST, NAOC, Chinese Academy of Sciences, 100012 Beijing, People's Republic of China \\ ${ }^{4}$ Korea Astronomy and Space Science Institute, 776 Daedeokdae-ro, Yuseong-gu, Daejeon 34055, Republic of Korea \\ ${ }^{5}$ East Asian Observatory, 660 N. A'ohōkū Place, Hilo, HI 96720-2700, USA \\ ${ }^{6}$ National Astronomical Observatory of Japan, National Institutes of Natural Sciences, 2-21-1 Osawa, Mitaka, Tokyo 181-8588, Japan \\ ${ }^{7}$ IAPS-INAF, via Fosso del Cavaliere, 100, I-00133 Roma, Italy \\ ${ }^{8}$ University Observatory Munich, Scheinerstrasse 1, D-81679 Munich, Germany \\ ${ }^{9}$ University of Chinese Academy of Sciences, Beijing 100049, People's Republic of China \\ ${ }^{10}$ European Southern Observatory, Karl-Schwarzschild-Str.2, D-85748 Garching bei München, Germany \\ ${ }^{11}$ Korea University of Science \& Technology, 176 Gajeong-dong, Yuseong-gu, Daejeon, Republic of Korea \\ ${ }^{12}$ Graduate Institute of Astronomy, National Central University 300, Jhongli City, Taoyuan County 32001, Taiwan \\ ${ }^{13}$ Astrophysics Research Institute, Liverpool John Moores University, IC2, Liverpool Science Park, 146 Brownlow Hill, Liverpool, L3 5RF, UK \\ ${ }^{14}$ ESA/STScI, 3700 San Martin Dr., Baltimore, MD 21218, USA \\ ${ }^{15}$ Xinjiang Astronomical Observatory, CAS, 150, Science 1-street, 830011 Urumqi, People's Republic of China \\ ${ }^{16}$ Astronomy Department, University of California, Berkeley, CA 94720, USA \\ ${ }^{17}$ Academia Sinica, Institute of Astronomy and Astrophysics, P.O. Box 23-141, Taipei 106, Taiwan \\ ${ }^{18}$ Department of Astronomy, Peking University, 100871 Beijing, People's Republic of China \\ ${ }^{19}$ Department of Physics, P.O.Box 64, FI-00014, University of Helsinki, Finland \\ ${ }^{20}$ Physics Department, Nazarbayev University, Kabanbay batyr avenue 53, 010000 Astana, Kazakhstan \\ ${ }^{21}$ Jet Propulsion Laboratory, California Institute of Technology, 4800 Oak Grove Drive, Pasadena, CA 91109, USA \\ ${ }^{23}$ Konkoly Observatory of the Hungarian Academy of Sciences, H-1121 Budapest, Konkoly Thege Miklós út 15-17, Hungary \\ Received 2018 January 7; revised 2018 May 9; accepted 2018 May 9; published 2018 June 20
}

\begin{abstract}
In order to understand the initial conditions and early evolution of star formation in a wide range of Galactic environments, we carried out an investigation of 64 Planck Galactic cold clumps (PGCCs) in the second quadrant of the Milky Way. Using the ${ }^{13} \mathrm{CO}$ and $\mathrm{C}^{18} \mathrm{O} J=1-0$ lines and $850 \mu \mathrm{m}$ continuum observations, we investigated cloud fragmentation and evolution associated with star formation. We extracted 468 clumps and 117 cores from the ${ }^{13} \mathrm{CO}$ line and $850 \mu \mathrm{m}$ continuum maps, respectively. We made use of the Bayesian distance calculator and derived the distances of all 64 PGCCs. We found that in general, the mass-size plane follows a relation of $m \sim r^{1.67}$. At a given scale, the masses of our objects are around 1/10 of that of typical Galactic massive star-forming regions. Analysis of the clump and core masses, virial parameters, densities, and mass-size relation suggests that the PGCCs in our sample have a low core formation efficiency $(\sim 3.0 \%)$, and most PGCCs are likely low-mass star-forming candidates. Statistical study indicates that the $850 \mu \mathrm{m}$ cores are more turbulent, more optically thick, and denser than the ${ }^{13} \mathrm{CO}$ clumps for star formation candidates, suggesting that the $850 \mu \mathrm{m}$ cores are likely more appropriate future star formation candidates than the ${ }^{13} \mathrm{CO}$ clumps.
\end{abstract}

Key words: dust, extinction - ISM: clouds - ISM: structure - stars: formation

Supporting material: figure sets, machine-readable tables

\section{Introduction}

Stars form in the dense, cold regions within molecular clouds. However, the physical and chemical properties of the cold compact objects that breed stars are still poorly understood. Stars could form out of gravitationally bound substructures within a molecular cloud, but how the substructures themselves form is strongly debated (e.g., Johnstone et al. 2004). Investigating the cloud fragmentation from large scale to small scale may be one way to determine this. An important approach to improve our understanding is to perform a statistical study toward the cold dense clumps from unbiased large surveys in the Milky Way.

Fortunately, the Planck satellite has allowed for a systematically extracted inventory of Galactic cold clumps (Planck
Collaboration et al. 2011a) using multiple bands from submillimeter to millimeter wavelengths. The Cold Core Catalog of Planck Objects (C3PO), consisting of 10,783 cold cores (Planck Collaboration et al. 2011d), and the Planck Early Release Cold Cores Catalog (ECC), the subcatalog containing 915 of the most reliable detections, were released in 2011 (Planck Collaboration et al. 2011b). The C3PO was the first unbiased, all-sky catalog of cold objects; it gives an unprecedented statistical view of the properties of these potential prestellar clumps and offers a unique possibility for their classification in terms of their intrinsic properties and environment (Planck Collaboration et al. 2011d). The cores in C3PO have relatively high column densities $(0.1 \sim$ $\left.1.6 \times 10^{22} \mathrm{~cm}^{-2}\right)$ and low dust temperatures $(\sim 10-15 \mathrm{~K}$; Planck Collaboration et al. 2011c, 2011d). This was followed by the 
Planck catalog of Galactic cold clumps (PGCCs; Planck Collaboration et al. 2016), an all-sky catalog of Galactic cold clump candidates containing 13,188 Galactic sources detected by Planck. This catalog is the full version of the ECC catalog. The Herschel key program "Galactic Cold Cores" was a follow-up to study the substructure and physics of selected C3PO sources (selection being performed on their intrinsic properties and Galactic location). This study commenced during the Herschel Science Demonstration Phase Data (Juvela et al. 2010).

Further follow-up studies of PGCC objects have been carried out with ground-based telescopes to study the evolutionary conditions of PGCCs. These facilities include the James Clerk Maxwell Telescope (JCMT), the Purple Mountain Observatory (PMO), the Nobeyama Radio Observatory, the Taeduk Radio Astronomy Observatory (TRAO), the Korean VLBI Network $(\mathrm{KVN})$, the Caltech Submillimeter Observatory (CSO), the Submillimeter Array (SMA), and the Institut de radioastronomie millimétrique (IRAM; Liu et al. 2012, 2013, 2015, 2016; Wu et al. 2012; Meng et al. 2013; Yuan et al. 2016; Zhang et al. 2016b; Kim et al. 2017; Tatematsu et al. 2017; Tang et al. 2018; Yi et al. 2018). These ground-based studies allow us to improve our understanding of dense cores and star formation in widely different environments at higher spatial resolution than the Planck observations, using different tracers from the continuum to spectral lines (e.g., $\mathrm{CO}, \mathrm{N}_{2} \mathrm{H}^{+}, \mathrm{HCO}^{+}$). For example, Wu et al. (2012) and Meng et al. (2013) carried out a survey toward 745 PGCCs in ${ }^{12} \mathrm{CO},{ }^{13} \mathrm{CO}$, and $\mathrm{C}^{18} \mathrm{O}$ $J=1-0$ using the PMO $13.7 \mathrm{~m}$ telescope. They found a variety of morphologies from extended diffuse to dense, isolated, cometary, and filamentary structures. They also found that the PGCCs are the most quiescent among the sample of weak-red Infrared Astronomical Satellite (IRAS), infrared-dark clouds, UC H II candidates, extended green objects, and methanol maser sources. Liu et al. (2016) performed a series of observations with ground-based telescopes toward one PGCC in the $\lambda$ Orionis complex to systematically investigate the effects of stellar feedback. Particularly, they discovered an extremely young Class 0 protostellar object (G192N) and a proto-brown dwarf candidate (G192S) located in a gravitationally bound bright-rimmed clump. This provides a sample to study the earliest stage of star formation. Yuan et al. (2016) conducted the first large survey of dense gas toward PGCCs in the $J=1-0$ transitions of $\mathrm{HCO}^{+}$and $\mathrm{HCN}$ toward 621 molecular cores. On the basis of an inspection of the derived density information given in their PGCC catalog, Yuan et al. (2016) suggested that about 1000 out of 13,188 PGCCs show a sufficient reservoir of dense gas to form stars.

Based on the studies mentioned above, PGCCs are cold $(\sim 10-15 \mathrm{~K})$ and turbulence-dominated and have relatively low column densities compared to other star-forming regions (Planck Collaboration et al. 2011c, 2011d; Wu et al. 2012). Additionally, most clumps are quiescent and lack signs of star formation, indicating that the PGCCs are most likely in the very initial evolutionary stages of star formation (Wu et al. 2012; Yuan et al. 2016). Furthermore, previous studies indicate that gaseous $\mathrm{CO}$ abundance (or depletion) can be used as a tracer for the evolution of molecular clouds (Liu et al. 2013; Zhang et al. 2017a).

The work described here is part of the TOP-SCOPE ${ }^{24}$ survey of PGCCs, which combines the TRAO $13.7 \mathrm{~m}$ telescope and

\footnotetext{
24 TOP: TRAO observations of Planck cold clumps; SCOPE: SCUBA-2 Continuum Observations of Pre-protostellar Evolution.
}

the Submillimetre Common-User Bolometer Array 2 (SCUBA2; Holland et al. 2013) instrument on the JCMT to observe around 1000 PGCCs (D. Eden et al. 2018, in preparation; Liu et al. 2018a). It is a follow-up study of Zhang et al. (2016b), who mainly used ${ }^{12} \mathrm{CO}$ and ${ }^{13} \mathrm{CO} J=1-0$ emission lines to investigate the gas content of 96 PGCCs from $98^{\circ}<l<180^{\circ}$ and $-4^{\circ}<b<10^{\circ}$ in the second quadrant of the Milky Way. The survey has covered most of the densest ECCs in the regions of the second quadrant (see more details in Zhang et al. 2016b). Zhang et al. (2016b) discussed the properties and morphologies of these clumps, combining the distributions of excitation temperature, velocity dispersion, and column density. The second quadrant is home to many well-known star formation regions, such as W3, W4, W5, NGC 7129, NGC 7538, and S235 (Dame et al. 1987, 2001; Heyer \& Terebey 1998). A systematic cold core analysis of the second quadrant could thus be essential for understanding the properties of the initial star-forming conditions in the outer Galaxy.

In the 96 Zhang et al. (2016b) PGCCs, there are 64 sources that have been covered by both the SCUBA-2 $850 \mu \mathrm{m}$ continuum and $\mathrm{PMO}{ }^{13} \mathrm{CO}$ and $\mathrm{C}^{18} \mathrm{O} J=1-0$ line observations. In this work, we study the 64 PGCCs, mainly combining the continuum and line data mentioned above. The ${ }^{13} \mathrm{CO}$ and $\mathrm{C}^{18} \mathrm{O}$ are more suitable tracers to study the dense conditions of the PGCCs than the ${ }^{12} \mathrm{CO}$ and ${ }^{13} \mathrm{CO}$ investigation in Zhang et al. (2016b). These data are also compared with the WISE 12 and $22 \mu \mathrm{m}$ emission. The full sample is presented in Table 1 and Figure 1. Section 2 presents the observations and data reduction. Section 3 shows the results of observations and data analysis. In Section 4, we discuss the fragmentation and evolution associated with star formation and present a statistical analysis of the morphology, velocity dispersion, virial parameter, surface density, optical depth, and excitation temperature for the ${ }^{13} \mathrm{CO}$ clumps and $850 \mu \mathrm{m}$ cores. Finally, a summary is presented in Section 5.

\section{Observations}

\subsection{The CO Data of the PMO $13.7 \mathrm{~m}$ Telescope}

The CO observations were made during 2011 April-May and 2011 December-2012 January using the $13.7 \mathrm{~m}$ millimeter telescope of Qinghai Station at the PMO. ${ }^{25}$ The nine-beam SIS superconducting receiver with beams separated by around $180^{\prime \prime}$ was used as the front end. The receiver was operated in the sideband separation of single sideband mode, allowing for simultaneous observations of three $\mathrm{CO} J=1-0$ isotopologues, with ${ }^{12} \mathrm{CO}$ in the upper sideband (USB) and ${ }^{13} \mathrm{CO}$ and $\mathrm{C}^{18} \mathrm{O}$ in the lower sideband (LSB). The half-power beam width (HPBW) is $52^{\prime \prime} \pm 3^{\prime \prime}$, with a main-beam efficiency of $\sim 50 \%$ for ${ }^{13} \mathrm{CO}$ and $\mathrm{C}^{18} \mathrm{O}$ observations. The ${ }^{13} \mathrm{CO}$ and $\mathrm{C}^{18} \mathrm{O}$ data are used here. The pointing and tracking accuracies are better than $5^{\prime \prime}$. The typical system temperature during the runs was $\sim 120 \mathrm{~K}$ at $110.2 \mathrm{GHz}$ and varied by $\sim 10 \%$ between beams. A fast Fourier transform (FFT) spectrometer was used as the back end with a total bandwidth of $1 \mathrm{GHz}$ and 16,384 channels, giving a velocity resolution of $\sim 0.16 \mathrm{~km} \mathrm{~s}^{-1}$ for the ${ }^{13} \mathrm{CO}$ and $\mathrm{C}^{18} \mathrm{O}$ lines.

An on-the-fly (OTF) observing mode was used for the mapping observations at a scan speed of $50^{\prime \prime} \mathrm{s}^{-1}$. The off position for each "off" source was carefully chosen from an

\footnotetext{
25 http://www.dlh.pmo.cas.cn/
} 
Table 1

64 Planck Cold Clumps in the Second Quadrant

\begin{tabular}{|c|c|c|c|c|}
\hline Name & $\begin{array}{c}\text { R.A.(J2000) } \\
\text { hh:mm:ss }\end{array}$ & $\begin{array}{c}\text { Decl.(J2000) } \\
\text { dd:mm:ss }\end{array}$ & $\begin{array}{c}\text { Distance } \\
\mathrm{kpc}\end{array}$ & Prob. ${ }^{\mathrm{a}}$ \\
\hline G098.50-03.24 & $22: 05: 00.08$ & $+51: 33: 11.69$ & $1.59(0.25)$ & 0.76 \\
\hline G108.85-00.80 & $22: 58: 51.53$ & $+58: 57: 27.09$ & $3.21(0.38)$ & 0.56 \\
\hline G110.65+09.65 & $22: 28: 00.22$ & $+69: 01: 48.10$ & $0.82(0.11)$ & 1.00 \\
\hline G112.52+08.38 & $22: 52: 47.62$ & $+68: 49: 28.31$ & $0.78(0.10)$ & 1.00 \\
\hline G112.60+08.53 & $22: 52: 54.76$ & $+68: 59: 53.90$ & $0.78(0.10)$ & 1.00 \\
\hline G115.92+09.46 & $23: 24: 04.62$ & $+71: 08: 08.69$ & $0.73(0.08)$ & 1.00 \\
\hline G116.08-02.38 & $23: 56: 41.79$ & $+59: 45: 13.19$ & $0.72(0.09)$ & 1.00 \\
\hline G116.12+08.98 & $23: 28: 14.03$ & $+70: 45: 12.38$ & $0.73(0.08)$ & 1.00 \\
\hline G120.16+03.09 & $00: 24: 26.01$ & $+65: 49: 27.59$ & $1.28(0.65)$ & 0.88 \\
\hline G120.67+02.66 & $00: 29: 41.95$ & $+65: 26: 39.99$ & $0.90(0.29)$ & 0.97 \\
\hline G120.98+02.66 & $00: 32: 38.94$ & $+65: 28: 07.08$ & $0.92(0.04)$ & 1.00 \\
\hline G121.35+03.39 & $00: 35: 48.66$ & $+66: 13: 13.29$ & $0.70(0.08)$ & 1.00 \\
\hline G121.90-01.54 & $00: 42: 52.64$ & $+61: 18: 23.20$ & $0.59(0.20)$ & 0.91 \\
\hline G121.92-01.71 & $00: 43: 06.34$ & $+61: 08: 21.59$ & $0.58(0.20)$ & 0.91 \\
\hline G125.66-00.55 & $01: 14: 52.20$ & $+62: 11: 16.60$ & $0.61(0.16)$ & 0.53 \\
\hline G126.49-01.30 & $01: 21: 14.55$ & $+61: 21: 34.60$ & $0.93(0.15)$ & 0.57 \\
\hline G126.95-01.06 & $01: 25: 19.48$ & $+61: 32: 36.19$ & $0.60(0.17)$ & 0.52 \\
\hline G127.22-02.25 & $01: 26: 10.18$ & $+60: 19: 29.30$ & $0.88(0.19)$ & 1.00 \\
\hline G127.88+02.66 & 01:38:39.10 & $+65: 05: 06.49$ & $0.89(0.11)$ & 1.00 \\
\hline G128.95-00.18 & $01: 43: 15.17$ & $+62: 04: 39.09$ & $0.92(0.18)$ & 0.59 \\
\hline $\mathrm{G} 131.72+09.70$ & $02: 39: 57.51$ & $+70: 42: 11.60$ & $0.57(0.16)$ & 1.00 \\
\hline $\mathrm{G} 132.07+08.80$ & $02: 39: 18.17$ & $+69: 44: 01.11$ & $0.59(0.15)$ & 1.00 \\
\hline G132.03+08.95 & $02: 39: 33.56$ & $+69: 53: 21.08$ & $0.59(0.15)$ & 1.00 \\
\hline G133.28+08.81 & $02: 51: 42.22$ & $+69: 14: 13.39$ & $0.58(0.15)$ & 1.00 \\
\hline G133.48+09.02 & $02: 54: 44.50$ & $+69: 19: 57.59$ & $0.61(0.14)$ & 0.62 \\
\hline G136.31-01.77 & 02:36:07.02 & $+58: 21: 09.09$ & $0.51(0.17)$ & 1.00 \\
\hline G140.49+06.07 & $03: 37: 46.12$ & $+63: 07: 27.29$ & $1.23(0.49)$ & 0.63 \\
\hline $\mathrm{G} 140.77+05.00$ & $03: 34: 18.18$ & $+62: 05: 35.89$ & $0.56(0.14)$ & 0.74 \\
\hline G142.49+07.48 & $03: 59: 13.56$ & $+62: 58: 52.40$ & $0.55(0.14)$ & 0.79 \\
\hline G142.62+07.29 & 03:59:00.66 & $+62: 45: 12.60$ & $0.54(0.14)$ & 1.00 \\
\hline $\mathrm{G} 144.84+00.76$ & $03: 40: 20.80$ & $+56: 16: 28.09$ & $2.20(0.28)$ & 1.00 \\
\hline $\mathrm{G} 146.11+07.80$ & $04: 23: 14.52$ & $+60: 44: 31.20$ & $0.52(0.14)$ & 1.00 \\
\hline $\mathrm{G} 146.71+02.05$ & $03: 56: 37.16$ & $+56: 07: 23.10$ & $0.47(0.15)$ & 1.00 \\
\hline G147.01+03.39 & 04:04:41.36 & $+56: 56: 16.79$ & $0.50(0.14)$ & 1.00 \\
\hline G148.00+00.09 & 03:54:48.04 & $+53: 47: 19.89$ & $2.15(0.28)$ & 0.83 \\
\hline G148.24+00.41 & $03: 57: 26.18$ & $+53: 52: 36.30$ & $2.17(0.29)$ & 0.77 \\
\hline G149.23+03.07 & $04: 14: 48.52$ & $+55: 12: 03.29$ & $0.47(0.15)$ & 1.00 \\
\hline G149.41+03.37 & 04:17:09.06 & $+55: 17: 39.39$ & $0.47(0.15)$ & 1.00 \\
\hline G149.52-01.23 & $03: 56: 52.61$ & $+51: 48: 01.70$ & $0.51(0.14)$ & 0.87 \\
\hline G149.58+03.45 & $04: 18: 23.93$ & $+55: 13: 30.59$ & $0.47(0.15)$ & 1.00 \\
\hline G149.65+03.54 & $04: 19: 11.24$ & $+55: 14: 44.39$ & $0.47(0.15)$ & 1.00 \\
\hline G150.22+03.91 & $04: 23: 51.69$ & $+55: 06: 22.50$ & $0.46(0.14)$ & 1.00 \\
\hline G150.44+03.95 & 04:25:07.08 & $+54: 58: 32.39$ & $0.46(0.14)$ & 1.00 \\
\hline $\mathrm{G} 151.08+04.46$ & $04: 30: 42.87$ & $+54: 51: 53.89$ & $0.46(0.14)$ & 1.00 \\
\hline G151.45+03.95 & $04: 29: 56.25$ & $+54: 14: 51.70$ & $0.46(0.14)$ & 1.00 \\
\hline G154.90+04.61 & $04: 48: 27.03$ & $+52: 06: 30.39$ & $0.45(0.14)$ & 1.00 \\
\hline G156.04+06.03 & 05:00:19.24 & $+52: 06: 45.60$ & $0.42(0.13)$ & 1.00 \\
\hline G156.20+05.26 & $04: 57: 00.65$ & $+51: 31: 08.89$ & $0.44(0.14)$ & 1.00 \\
\hline G157.25-01.00 & 04:32:09.45 & $+46: 37: 25.00$ & $0.46(0.14)$ & 1.00 \\
\hline G159.52+03.26 & $04: 59: 55.05$ & $+47: 40: 52.20$ & $1.97(0.35)$ & 0.82 \\
\hline $\mathrm{G} 162.79+01.34$ & $05: 02: 42.87$ & $+43: 55: 05.70$ & $0.45(0.14)$ & 0.83 \\
\hline G169.14-01.13 & $05: 12: 20.07$ & $+37: 20: 57.09$ & $1.87(0.23)$ & 0.92 \\
\hline $\mathrm{G} 171.03+02.66$ & $05: 33: 35.43$ & $+37: 56: 42.69$ & $1.82(0.22)$ & 0.81 \\
\hline G171.34+02.59 & 05:34:06.95 & $+37: 38: 47.30$ & $1.78(0.20)$ & 0.80 \\
\hline $\mathrm{G} 172.85+02.27$ & $05: 36: 51.80$ & $+36: 11: 58.29$ & $1.71(0.13)$ & 0.77 \\
\hline $\mathrm{G} 175.20+01.28$ & $05: 38: 55.10$ & $+33: 41: 05.89$ & $1.69(0.12)$ & 0.78 \\
\hline $\mathrm{G} 175.53+01.34$ & $05: 39: 59.24$ & $+33: 26: 08.80$ & $1.69(0.12)$ & 0.75 \\
\hline G176.17-02.10 & $05: 27: 55.18$ & $+31: 01: 34.99$ & $4.96(0.44)$ & 0.39 \\
\hline G176.35+01.92 & $05: 44: 23.17$ & $+33: 02: 58.99$ & $1.70(0.13)$ & 0.63 \\
\hline G176.94+04.63 & 05:57:00.77 & $+33: 55: 16.30$ & $1.78(0.22)$ & 0.53 \\
\hline $\mathrm{G} 177.09+02.85$ & $05: 50: 02.12$ & $+32: 53: 35.90$ & $4.97(0.57)$ & 0.49 \\
\hline G177.14-01.21 & $05: 33: 52.82$ & $+30: 42: 36.29$ & $5.00(0.48)$ & 0.46 \\
\hline $\mathrm{G} 177.86+01.04$ & $05: 44: 35.76$ & $+31: 17: 57.40$ & $4.99(0.64)$ & 0.53 \\
\hline
\end{tabular}

Table 1

(Continued)

\begin{tabular}{lcccc}
\hline \hline Name & $\begin{array}{c}\text { R.A.(J2000) } \\
\text { hh:mm:ss }\end{array}$ & $\begin{array}{c}\text { Decl.(J2000) } \\
\text { dd:mm:ss }\end{array}$ & $\begin{array}{c}\text { Distance } \\
\text { kpc }\end{array}$ & Prob. $^{{ }^{a}}$ \\
\hline G178.28-00.61 & $05: 39: 03.83$ & $+30: 04: 05.90$ & $0.96(0.02)$ & 0.36 \\
\hline
\end{tabular}

Note.

${ }^{\mathrm{a}}$ The distance probabilities derived by the Bayesian distance calculator.

(This table is available in machine-readable form.)

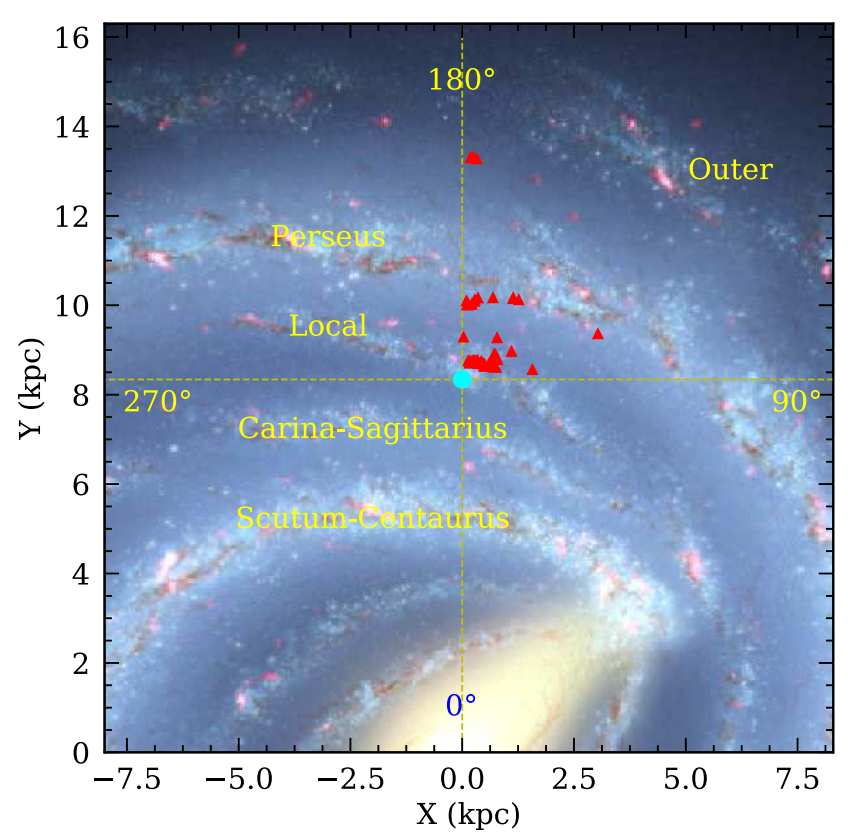

Figure 1. Distribution of the clumps (red filled triangles) on the background of an artist's conception of the Milky Way (R. Hurt: NASA/JPL-Caltech/SSC). All sources are located in the second quadrant of the Galaxy.

area within a $3^{\circ}$ radius of each "on" source, where there is extremely weak or no CO emission (Dame et al. 1987, 2001). The antenna continuously scanned a region of $22^{\prime} \times 22^{\prime}$ centered on each clump, while only the central $14^{\prime} \times 14^{\prime}$ region was used due to the noisy edges of the OTF maps. The rms noise level was $0.1 \mathrm{~K}$ in the main-beam antenna temperature $T_{\mathrm{A}}^{*}$ for ${ }^{13} \mathrm{CO}$ and $\mathrm{C}^{18} \mathrm{O} J=1-0$. The OTF data were resampled in a three-dimensional (3D) cube with a grid spacing of $30^{\prime \prime}$. The IRAM software package GILDAS ${ }^{26}$ was used for the data reduction. The reduced images are presented in Figures 2 and 3. The integrated-intensity maps of the ${ }^{13} \mathrm{CO}$ line are also overlaid on the WISE 12 and $22 \mu \mathrm{m}$ emission maps in Figures 4 and 5.

\subsection{The $850 \mu \mathrm{m}$ Data of the JCMT $15 \mathrm{~m}$ Telescope}

The majority of the SCUBA-2 $850 \mu \mathrm{m}$ observations were conducted as part of the SCOPE project (Liu et al. 2018a). The rest of the data were collected from the JCMT data archive of the Canadian Astronomy Data Centre (CADC). SCUBA-2 is a bolometer detector at the JCMT $15 \mathrm{~m}$ telescope with $\sim 10,000$ pixels over eight science arrays that simultaneously observe 450 and $850 \mu \mathrm{m}$ with a field of view of $8^{\prime}$, and the effective beam size is around $10^{\prime \prime}$ at $450 \mu \mathrm{m}$ and $14^{\prime \prime}$ at $850 \mu \mathrm{m}$ (Holland

\footnotetext{
${ }^{26}$ http://iram.fr/IRAMFR/GILDAS/
} 

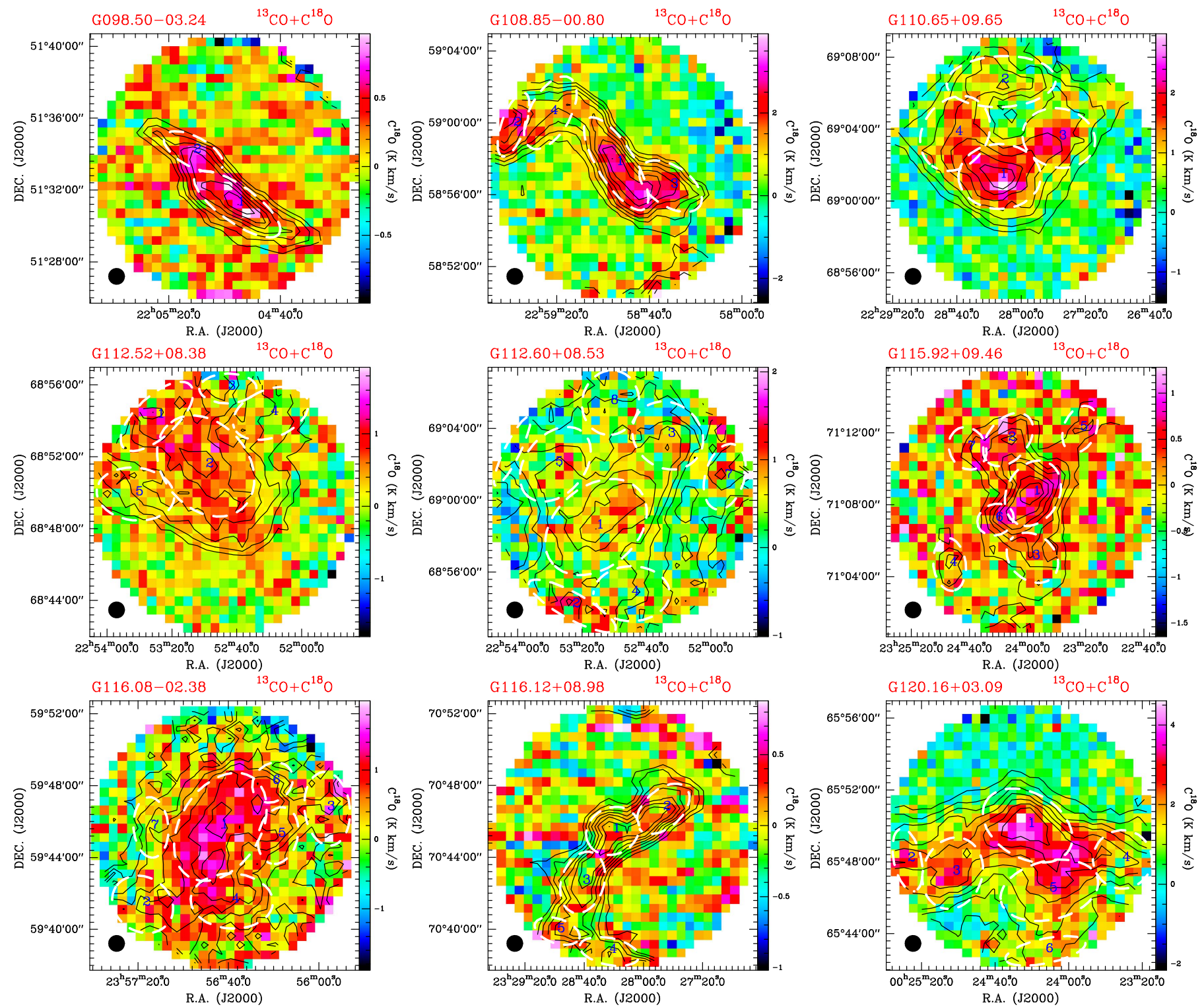

Figure 2. Integrated-intensity maps of the $\mathrm{C}^{18} \mathrm{O}$ emission of each source with overlaid contours of the ${ }^{13} \mathrm{CO}$ line. The integrated velocity ranges used are indicated within the red window in the corresponding spectrum of Figure 3 . The contour levels of the ${ }^{13} \mathrm{CO}$ lines are drawn at $10 \%$ steps, starting with $30 \%$ of the peak value. The white ellipses indicate the extracted ${ }^{13} \mathrm{CO}$ clumps. The beam size of the ${ }^{13} \mathrm{CO}$ data is indicated in the bottom left corner.

(The complete figure set (64 images) is available.)

et al. 2013). The observations used the constant velocity (CV) Daisy mode (Bintley et al. 2014), which is more sensitive in the central $3^{\prime}$ radii and designed for small and compact sources. The $225 \mathrm{GHz}$ opacity during the observations was in the range of $0.09-0.11$; therefore, we only use the $850 \mu \mathrm{m}$ data, as the $450 \mu \mathrm{m}$ data are not photometric. The data were reduced using SMURF in the STARLINK package (Chapin et al. 2013; Dempsey et al. 2013). The mapped areas were about $12^{\prime} \times 12^{\prime}$. The rms level in the central $3^{\prime}$ area of the maps was typically 6-10 $\mathrm{mJy}_{\text {beam }}{ }^{-1}$. The images are presented in Figure 6.

SCUBA-2 continuum observations at $850 \mu \mathrm{m}$ are known to be affected by contamination from spectral lines (Johnstone et al. 2003; Parsons et al. 2018), especially the ${ }^{12} \mathrm{CO} J=3-2$ line at $345.796 \mathrm{GHz}$ (Drabek et al. 2012). A typical level of the CO contamination is $<20 \%$ (Nutter \& Ward-Thompson 2007; Buckle et al. 2015; Moore et al. 2015; Rumble et al. 2015), which is not significant. In a study of 90 PGCCs, Juvela et al.
(2018) found that the CO contamination levels in the SCUBA2 images are $\leqslant 5 \%$. Therefore, we do not correct for it here.

The observatory-produced flux conversion factor (FCF) was calculated using a $60^{\prime \prime}$ aperture. If we have a clump that is bigger or smaller than this nominal FCF, we will need to adjust the flux values accordingly. The integrated flux density $S_{850 \mu \mathrm{m}}$ of each extracted core (see Section 3.2) has been corrected using the aperture correction factor provided by Dempsey et al. (2013).

\subsection{Archival WISE Data}

NASA's Wide-field Infrared Survey Explorer (WISE; Wright et al. 2010) mapped the sky at 3.4, 4.6, 12, and $22 \mu \mathrm{m}$ (W1, W2, W3, and W4) with an angular resolution of 6!" 1, 6!" 4, 6!"5, and 12 " 0 in the four bands, respectively. WISE achieved $5 \sigma$ point-source sensitivities better than $0.08,0.11,1$, and $6 \mathrm{mJy}$ in 

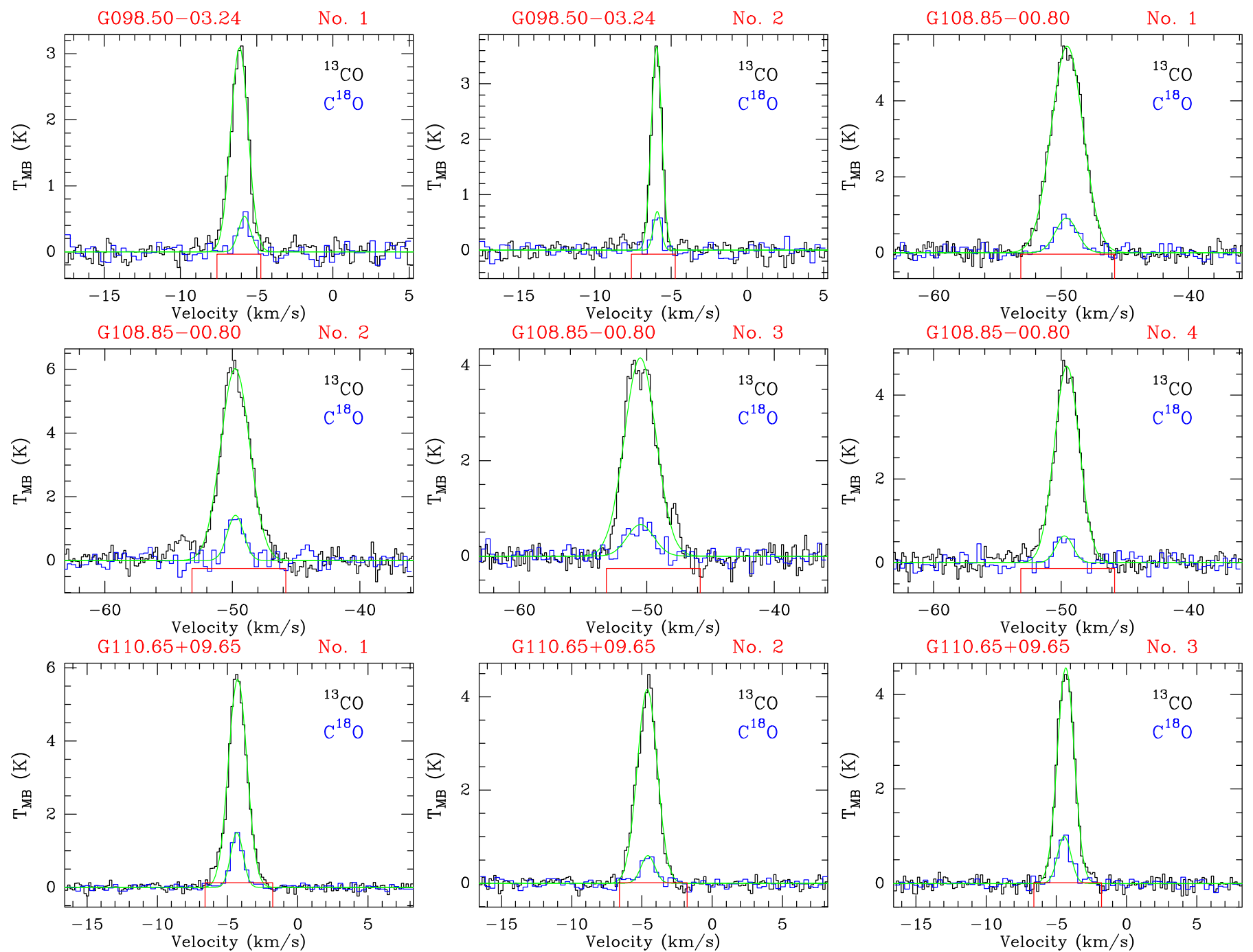

Figure 3. Averaged ${ }^{13} \mathrm{CO}$ (black line) and $\mathrm{C}^{18} \mathrm{O}$ (blue line) lines within the size of each extracted ${ }^{13} \mathrm{CO}$ clump (see Figure 2). The green lines show the Gaussian fits in each spectrum. The red window indicates the velocity range corresponding to the ${ }^{13} \mathrm{CO}$ and $\mathrm{C}^{18} \mathrm{O}$ integrated-intensity maps (see Figure 2 ).

(The complete figure set (468 images) is available.)

unconfused regions on the ecliptic in the four bands. The sensitivity was better toward the ecliptic poles due to denser coverage and lower zodiacal background. In this work, WISE 12 and $22 \mu \mathrm{m}$ image data are used. Additionally, the AllWISE Data in the VizieR Online Data Catalog (Cutri et al. 2013, 2014) are used for point-source cross-identification (within $10^{\prime \prime}$ radii of the peak position of each $850 \mu \mathrm{m}$ core) with our $850 \mu \mathrm{m}$ catalog (using the Gaussclumps procedure; see Section 3.2) listed in Table 5. The WISE images are presented in Figures 4 and 5.

\section{Results and Analysis}

\subsection{Distance Determination}

The distances to the PGCCs are estimated using the Bayesian distance calculator ${ }^{27}$ (Reid et al. 2016), which uses trigonometric parallaxes from the Bar and Spiral Structure Legacy Survey ${ }^{28}$ (BeSSeL) and Japanese VLBI Exploration

\footnotetext{
27 http://bessel.vlbi-astrometry.org/bayesian

$28 \mathrm{http}: / /$ bessel.vlbi-astrometry.org/home
}

of Radio Astrometry ${ }^{29}$ (VERA) projects to significantly improve the accuracy and reliability of kinematic distance estimates to other sources that are known to follow the Milky Way spiral structure. Based on the ${ }^{13} \mathrm{CO}$ centroid velocity of each $850 \mu \mathrm{m}$ core No. 1 within each PGCC (see Table 4), the corresponding distance parameters (distributed between 0.42 and $5.0 \mathrm{kpc}$ ) are derived and listed in Table 1. The probabilities of the adopted distances are also listed in Table 1. In Figure 1, we present the distribution of the PGCCs on an artist's conception of the Milky Way (Yuan et al. 2017). We find that most PGCCs are located in the Local and Perseus arms, with a significant population in the corresponding interarm region, while only four PGCCs (G176.17-02.10, G177.09+02.85, G177.14-01.21, and G177.86+01.04) are located in the Outer Arm. We note that some derived distances are different from those in Zhang et al. (2016b), who used only the Galactic rotation curve to acquire the kinematic distances (distribution between 0.1 and $28.7 \mathrm{kpc}$ ) following the method of Sofue (2011).

${ }^{29}$ http://veraserver.mtk.nao.ac.jp/ 

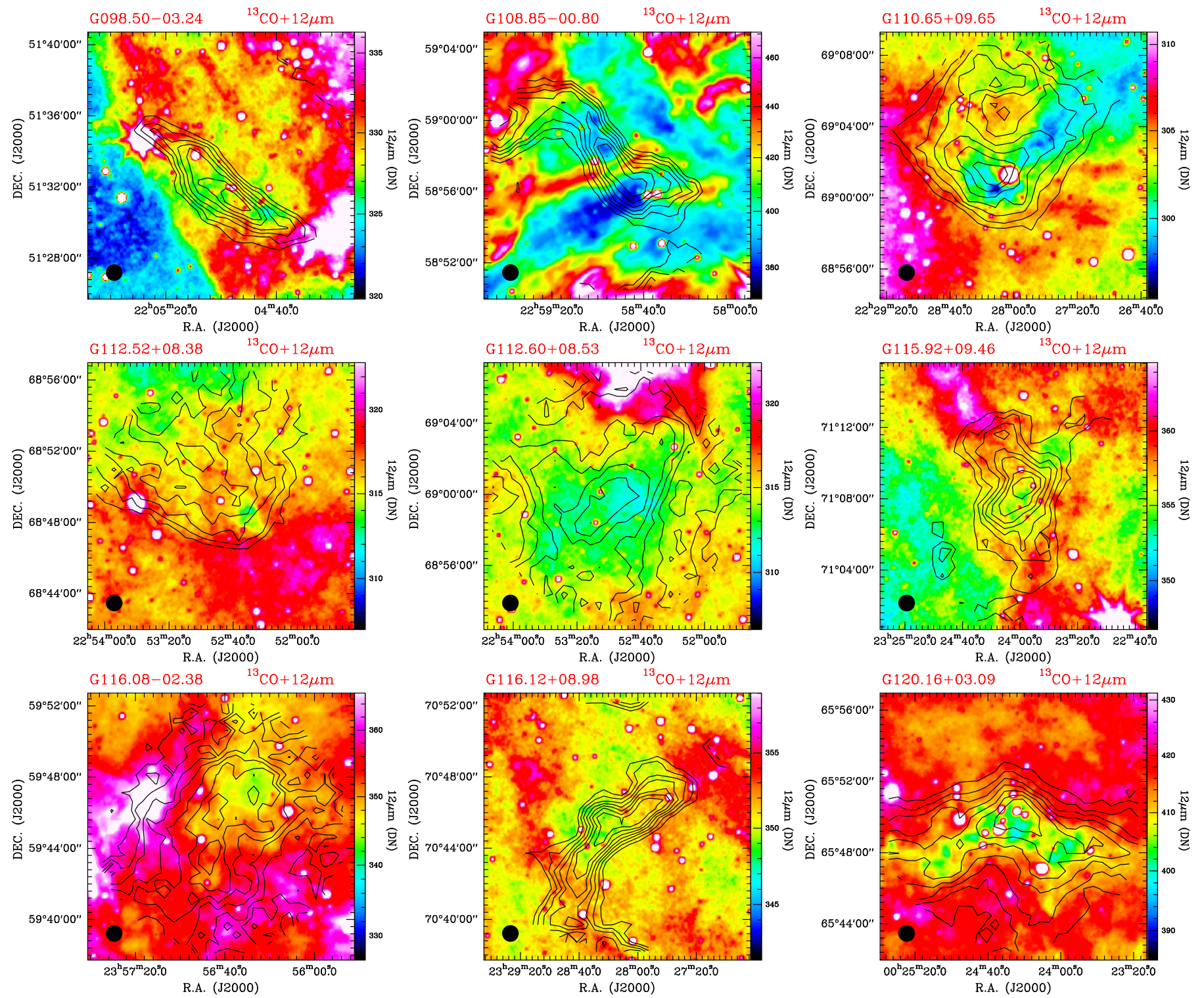

Figure 4. WISE $12 \mu \mathrm{m}$ emission for each PGCC with overlaid ${ }^{13} \mathrm{CO}$ contours. The contour levels of the ${ }^{13} \mathrm{CO}$ lines are drawn at $10 \%$ steps, starting with $30 \%$ of the peak value. The beam size of the ${ }^{13} \mathrm{CO}$ data is indicated in the bottom left corner.

(The complete figure set (64 images) is available.)

\subsection{Fragment Extraction and Definition}

The potential cloud fragments are extracted from the ${ }^{13} \mathrm{CO}$ integrated line intensity and $850 \mu \mathrm{m}$ continuum maps with the Gaussclumps procedure (Kramer et al. 1998; Stutzki \& Guesten 1990; Zhang et al. 2017b) in the GILDAS software package. Gaussclumps fits a two-dimensional fragment to the local maximum of the input cube; subtracts this fragment from the cube, creating a residual map; and then continues with the maximum of this residual map (Gómez et al. 2014). This procedure is then repeated until a stop criterion is met. We only consider fragments with peak ${ }^{13} \mathrm{CO}$ and $850 \mu \mathrm{m}$ intensities of above $5 \sigma$ with the initial FWHM set at 1.1 times the beam size. The initial aperture FWHM and aperture cutoff are set as 2.0 and 8.0 times the beam size, respectively (see also a detailed example of the configurations in Belloche et al. 2011). Considering that some extracted sources are in filamentary structures, we have rejected sources with aspect ratios larger than 5, as the study of filaments in the SCOPE PGCCs will be the subject of a further study. The measured parameters are listed in Tables 2-5 and indicated with ellipses in Figures 2 and 7.

In this work, we adopt "fragment" as the general name for both extracted clumps and cores. We consider a clump to have a typical size of $0.3-3 \mathrm{pc}$ with a mass of 50-500 $M_{\odot}$, and cores are an order of magnitude lower with sizes of $0.03-0.2 \mathrm{pc}$ with masses of $0.5-5 M_{\odot}$ (e.g., Bergin \& Tafalla 2007; Motte et al. 2017). Based on the effective radii in Tables 2 and 4, we thus refer to the ${ }^{13} \mathrm{CO}$ objects as clumps and the $850 \mu \mathrm{m}$ objects as cores. Massive clouds tend to fragment into clusters of clumps and cores (Pokhrel et al. 2018) in which young stars form. Therefore, we can explore the habitats of clumps at larger scales and cores at smaller scales, studying the fragmentation process. $^{30}$

\footnotetext{
${ }^{30}$ A caveat here is that the clumps and cores could just be discrete selfgravitating structures in a large-scale cloud (see also Section 3.6).
} 

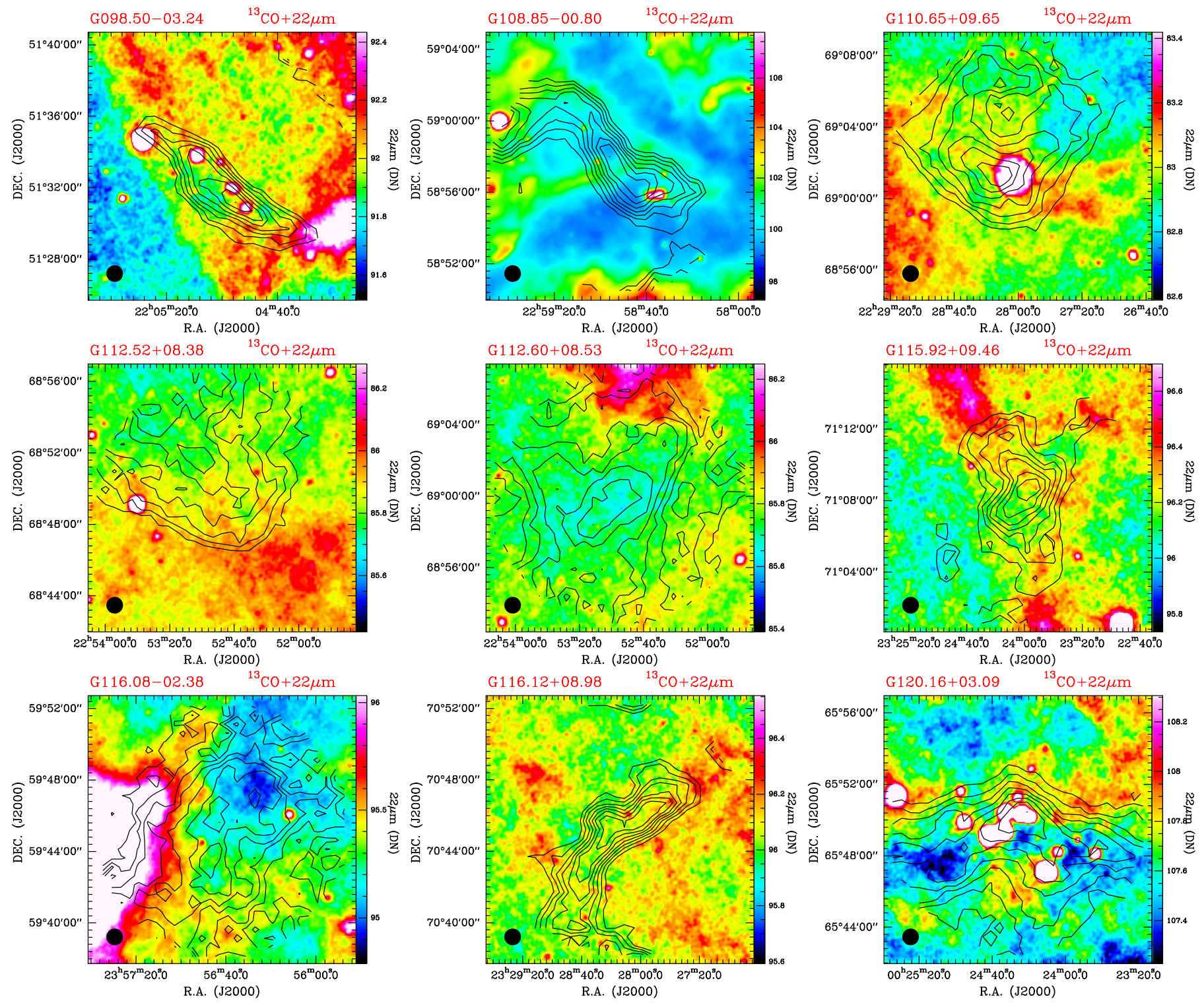

Figure 5. WISE $22 \mu \mathrm{m}$ emission for each PGCC with overlaid ${ }^{13} \mathrm{CO}$ contours. The contour levels of the ${ }^{13} \mathrm{CO}$ lines are drawn at $10 \%$ steps, starting with $30 \%$ of the peak value. The beam size of the ${ }^{13} \mathrm{CO}$ data is indicated in the bottom left corner.

(The complete figure set (64 images) is available.)

\section{3. ${ }^{13} \mathrm{CO}$ Clumps}

In total, we have extracted $468{ }^{13} \mathrm{CO}$ clumps having an effective radius range of $0.1-3.3 \mathrm{pc}$ with a median value of $0.4 \mathrm{pc}$ and a detected mass range of $1-6132 M_{\odot}$ with a median value of $66 M_{\odot}$ for the clumps. Figure 2 shows the $\mathrm{C}^{18} \mathrm{O}$ emission with ${ }^{13} \mathrm{CO}$ contours overlaid. Some ${ }^{13} \mathrm{CO}$ clumps have weak or no corresponding $\mathrm{C}^{18} \mathrm{O}$ emission. In Table 2, therefore, we only consider the sources that are detected in both of the lines with main-beam brightness temperatures $T^{1{ }^{3}} \mathrm{CO}>$ $3 \sigma$ and $T_{\mathrm{C}^{18} \mathrm{O}}>3 \sigma$. The white ellipses with numbers show the extracted ${ }^{13} \mathrm{CO}$ clumps. The average ${ }^{13} \mathrm{CO}$ and $\mathrm{C}^{18} \mathrm{O}$ lines within each extracted ${ }^{13} \mathrm{CO}$ clump are presented in Figure 3. We also display the Gaussian-fitted lines with green curves. Most of the ${ }^{13} \mathrm{CO}$ and $\mathrm{C}^{18} \mathrm{O}$ lines can be fitted with a singlevelocity Gaussian component. For the multi-velocity components, we only consider the strongest peak or the velocity components with infrared emission.
In Figure 2, morphologically, we observed that some PGCCs show clearly filamentary structure (e.g., G108.85-00.80, G116.12+08.98) and spherical structure (e.g., G115.92 +09.46 , G133.48+09.02), and the others are morphologically complicated and do not belong to both cases above. The filamentary structures are ubiquitous in the Milky Way (Rathborne et al. 2006; Csengeri et al. 2014; Motte et al. 2017; Zhang et al. 2017a). They are much elongated along the long axis of the filament with aspect ratios $\gtrsim 5$ (Wang et al. 2011, 2014). For the spherical structure, the most massive fragments are often located at the center position of their parent clusters, with several low-mass fragments surrounding the most massive one. We find that the filamentary structures make up $23(35.9 \%)$ sources in the 64 PGCCs, respectively. Dense clumps elongate along their parental filament axis. The clumps in filamentary structure seem to be more compact than the others in our sample. Könyves et al. (2015) suggested that the filamentary environment is more suitable for star formation 

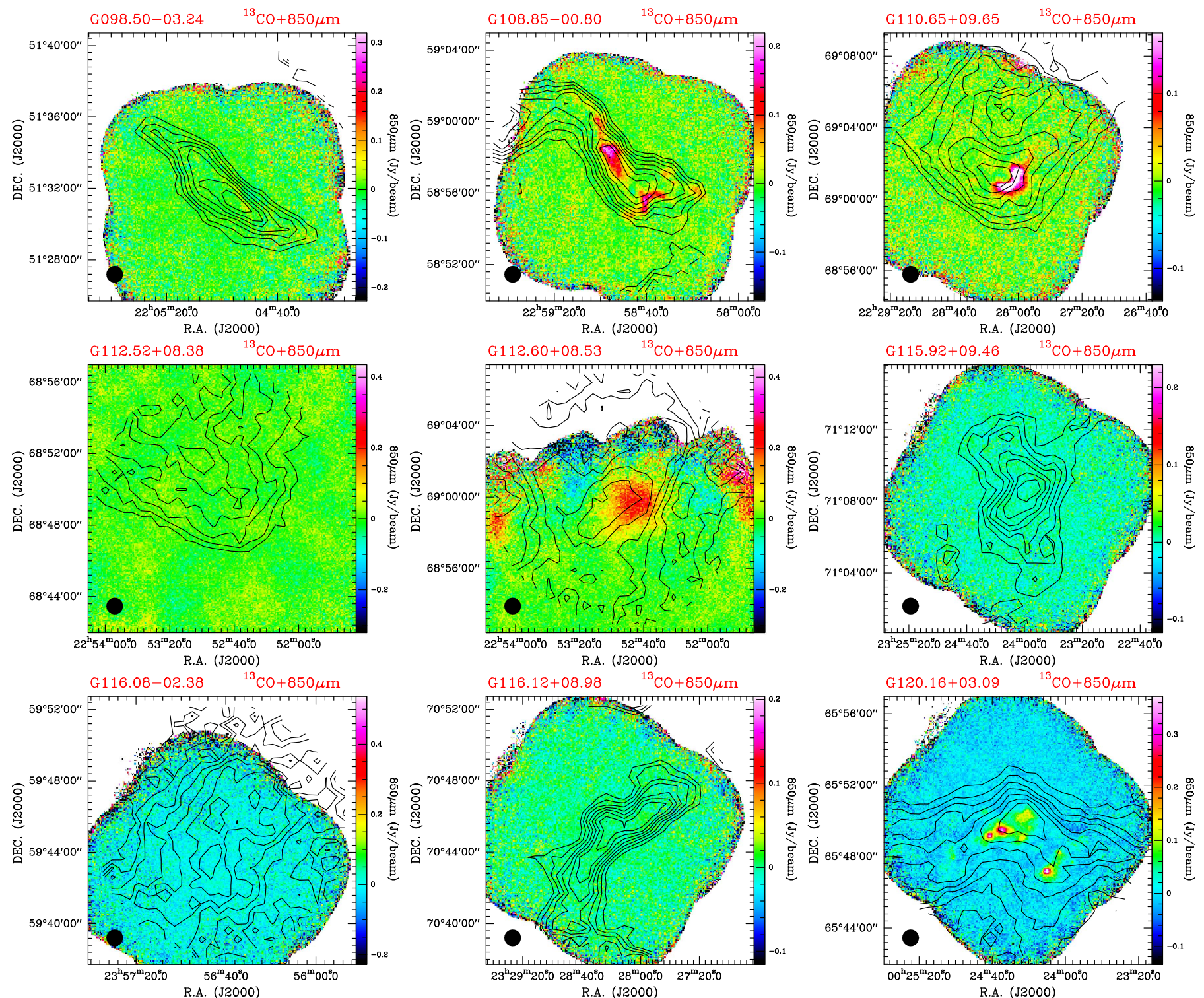

Figure 6. SCUBA-2 $850 \mu \mathrm{m}$ emission for each PGCC with overlaid ${ }^{13} \mathrm{CO}$ contours. The contour levels of the ${ }^{13} \mathrm{CO}$ lines are drawn at $10 \%$ steps, starting with $30 \%$ of the peak value. The beam size of the ${ }^{13} \mathrm{CO}$ data is indicated in the bottom left corner.

(The complete figure set (64 images) is available.)

than spherical structures. Lane et al. (2016) suggested that the dense core clusters tend to be elongated, perhaps indicating a formation mechanism linked to the filamentary structure within molecular clouds.

Figure 3 shows that only $56(12.0 \%)$ of the ${ }^{13} \mathrm{CO}$ clumps show multi-velocity components in both ${ }^{13} \mathrm{CO}$ and $\mathrm{C}^{18} \mathrm{O}$ emission, and the others have single-velocity components. In the direction of the second quadrant of the Milky Way, there are at most three spiral arms in the line of sight, and they are located at relatively near distances without kinematic distance ambiguity. The relatively optically thick ${ }^{13} \mathrm{CO}$ line has similar profiles to the optically thin $\mathrm{C}^{18} \mathrm{O}$ line for most clumps but shows a slightly broader width. Comparing the ${ }^{12} \mathrm{CO}$ line as a dynamical tracer with the ${ }^{13} \mathrm{CO}$ line, Zhang et al. (2016b) also found that clumps are mostly dynamically quiescent and lack star-forming activity, further indicating that the PGCCs are most likely in a very early evolutionary stage of star formation (Wu et al. 2012; Yuan et al. 2016).
In Figures 4 and $5,{ }^{13} \mathrm{CO}$ emission is plotted on maps of WISE 12 and $22 \mu \mathrm{m}$ emission. This is helpful for understanding the related infrared emission distribution in the background and to predict the interaction relationship between the ionized gas and molecular clouds (see details in Section 4.2).

\section{4. $850 \mu m$ Cores}

All 64 PGCCs have been observed at $850 \mu \mathrm{m}$ with SCUBA-2, but only $28(43.8 \%)$ are detected above $5 \sigma$ ( $\sigma$ is the rms noise of the image). The PGCCs G142.49+07.48 and G150.44 +03.95 are not adequately covered by the $850 \mu \mathrm{m}$ observations, which may reduce the detection number of $850 \mu \mathrm{m}$ cores but have no significant effect on the detection statistics. The low detection rate suggests that the PGCCs have a relatively low core formation efficiency (CFE; see Section 4.7). In total, we extracted $117850 \mu \mathrm{m}$ cores having an effective radius range of $0.03-0.48 \mathrm{pc}$ with a median value of $0.07 \mathrm{pc}$ and a detected 
Table 2

Observed Parameters of Extracted CO Clumps

\begin{tabular}{|c|c|c|c|c|c|c|c|c|c|}
\hline Name & $\begin{array}{l}\text { Offset (R.A. Decl.) } \\
\left({ }^{\mathrm{a}}{ }^{\prime \prime}\right)\end{array}$ & $\begin{array}{l}V_{13} \mathrm{CO}_{1} \\
\mathrm{~km} \mathrm{~s}^{-}\end{array}$ & $\begin{array}{l}\Delta V_{13} \mathrm{CO} \\
\mathrm{km} \mathrm{s}^{-1}\end{array}$ & $\begin{array}{c}T 13 \mathrm{CO} \\
\mathrm{K}\end{array}$ & $\begin{array}{c}V_{\mathrm{C}^{18} \mathrm{O}_{1}} \\
\mathrm{~km} \mathrm{~s}^{-1}\end{array}$ & $\begin{array}{l}\Delta V_{\mathrm{C}^{18} \mathrm{O}^{\mathrm{b}}} \\
\mathrm{km} \mathrm{s}^{-1}\end{array}$ & $\underset{\mathrm{K}}{T_{\mathrm{C}^{18} \mathrm{O}}}$ & $\tau{ }^{13} \mathrm{CO}^{\mathrm{c}}$ & $\begin{array}{c}T_{\mathrm{ex}}\left({ }^{13} \mathrm{CO}\right)^{\mathrm{d}} \\
\mathrm{K}\end{array}$ \\
\hline G098.50-03.24_1 & $-42.41,-118.79$ & $-6.12 \pm 0.01$ & $1.33 \pm 0.03$ & $3.09 \pm 0.11$ & $-5.80 \pm 0.07$ & $0.93 \pm 0.18$ & $0.54 \pm 0.10$ & $0.93 \pm 0.50$ & $10.11 \pm 1.80$ \\
\hline G098.50-03.24_2 & $103.25,56.95$ & $-5.95 \pm 0.01$ & $0.81 \pm 0.02$ & $3.67 \pm 0.12$ & $-5.91 \pm 0.04$ & $0.59 \pm 0.12$ & $0.70 \pm 0.09$ & $1.19 \pm 0.40$ & $11.10 \pm 1.07$ \\
\hline G108.85-00.80_1 & $21.64,16.41$ & $-49.53 \pm 0.01$ & $2.94 \pm 0.03$ & $5.44 \pm 0.15$ & $-49.59 \pm 0.08$ & $2.00 \pm 0.19$ & $0.91 \pm 0.12$ & $0.93 \pm 0.38$ & $16.93 \pm 2.35$ \\
\hline G108.85-00.80_2 & $364.31,146.82$ & $-49.78 \pm 0.02$ & $2.68 \pm 0.04$ & $6.02 \pm 0.24$ & $-49.75 \pm 0.07$ & $1.52 \pm 0.15$ & $1.42 \pm 0.20$ & $1.97 \pm 0.50$ & $15.46 \pm 0.85$ \\
\hline G108.85-00.80_3 & $-160.17,-58.88$ & $-50.51 \pm 0.02$ & $2.92 \pm 0.05$ & $4.16 \pm 0.19$ & $-50.49 \pm 0.14$ & $2.45 \pm 0.31$ & $0.66 \pm 0.15$ & $0.79 \pm 0.60$ & $14.10 \pm 3.94$ \\
\hline
\end{tabular}

$6 \quad$ Notes.

${ }^{a}$ The absolute coordinate of each source is listed in Table 1.

${ }^{\mathrm{b}}$ If the line width of $\mathrm{C}^{18} \mathrm{O}$ is less than two velocity channels (or two times the velocity resolution), the Gaussian fitting will produce a line width of $0.33 \pm 0.07 \mathrm{~km} \mathrm{~s}{ }^{-1}$.

${ }^{\mathrm{c}}$ The optical depth with $\tau^{13} \mathrm{CO}<0.20$ is unreliable; therefore, we set it as $\tau{ }^{13} \mathrm{CO}=0.20 \pm 0.05$.

${ }^{\mathrm{d}}$ The excitation temperature with $T_{\mathrm{ex}}\left({ }^{13} \mathrm{CO}\right)>50.00 \mathrm{~K}$ is unreliable; therefore, we set it as $T_{\mathrm{ex}}\left({ }^{13} \mathrm{CO}\right)=50.00 \pm 5.00 \mathrm{~K}$

(This table is available in its entirety in machine-readable form.) 

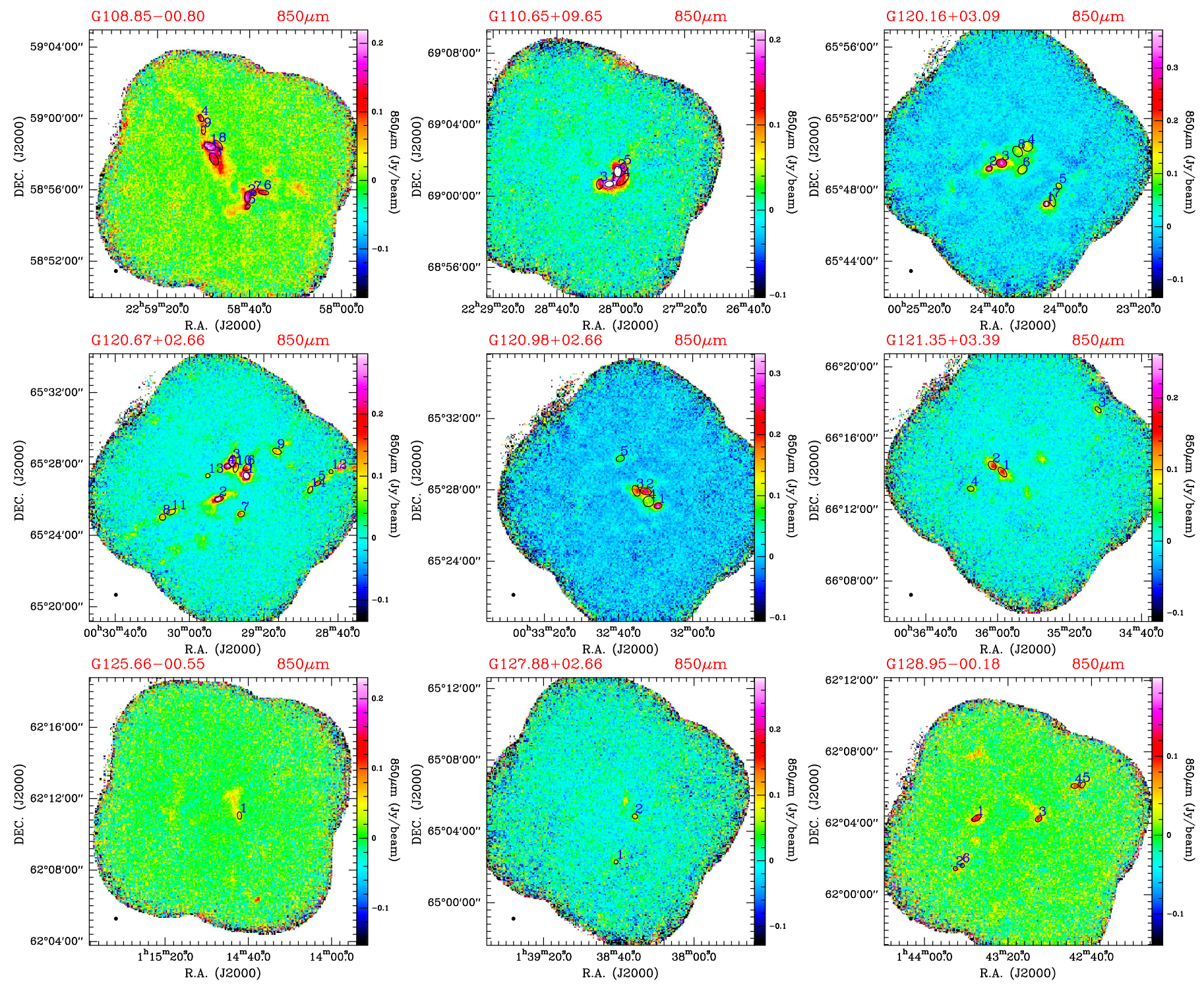

Figure 7. Extracted $850 \mu \mathrm{m}$ cores (black ellipse) superimposed on $850 \mu \mathrm{m}$ emission. The beam size of the $850 \mu \mathrm{m}$ data is indicated in the bottom left corner. (The complete figure set (28 images) is available.)

mass range of $0.4-311 M_{\odot}$ with a median value of $8 M_{\odot}$ for the cores. Figure 6 shows the $850 \mu \mathrm{m}$ emission map (color scale) with ${ }^{13} \mathrm{CO}$ contours overlaid. About $26(22.2 \%)$ of the 117 cores have weak $(<3 \sigma)$ or no corresponding $\mathrm{C}^{18} \mathrm{O}$ emission (see Figure 8 and Table 4 ). The $850 \mu \mathrm{m}$ cores are strongly associated with the peak positions of $\mathrm{C}^{18} \mathrm{O}$ emission.

In Table 5, we list the positional associations between our extracted $850 \mu \mathrm{m}$ cores and the AllWISE data (Cutri et al. $2013,2014)$. We only search for WISE point sources within $10^{\prime \prime}$ radii of the peak position of each $850 \mu \mathrm{m}$ core. In Figure 7, we show the distribution of the extracted $850 \mu \mathrm{m}$ cores. We find that $74(63.2 \%)$ of the $117850 \mu \mathrm{m}$ cores have corresponding WISE infrared point sources, some of which may happen to be the sources in the line of sight.

In Figure 8, we present the ${ }^{13} \mathrm{CO}$ and $\mathrm{C}^{18} \mathrm{O}$ lines extracted from the $117850 \mu \mathrm{m}$ cores. We find that only $26(22.2 \%)$ of the $850 \mu \mathrm{m}$ cores show multi-velocity components in the ${ }^{13} \mathrm{CO}$ and $\mathrm{C}^{18} \mathrm{O}$ lines, suggesting that most detections correspond to a single object along the line of sight. Compared with ${ }^{13} \mathrm{CO}$ clumps, the $850 \mu \mathrm{m}$ cores have few multi-peak spectra, indicating that majority ${ }^{13} \mathrm{CO}$ clumps at large scales are relatively more dynamically complex than the $850 \mu \mathrm{m}$ cores at small scales (see the error analysis in Section 4.1).

\subsection{Opacity, Excitation Temperature, Column Density, and Mass}

We use the approach of Wong et al. (2008) to derive the opacity, excitation temperature, and column density of each clump combining ${ }^{13} \mathrm{CO}$ and $\mathrm{C}^{18} \mathrm{O} J=1-0$. The integrated velocity ranges for each clump are shown in Figures 3 and 8. The relationship between opacities $(\tau)$ and main-beam brightness temperatures $\left(T_{\mathrm{MB}}\right)$ for ${ }^{13} \mathrm{CO}$ and $\mathrm{C}^{18} \mathrm{O}$ (Myers et al. 1983; Zhang \& Wang 2012; Sanhueza et al. 2012) is

$$
\frac{T_{\mathrm{MB}}\left({ }^{13} \mathrm{CO}\right)}{T_{\mathrm{MB}}\left(\mathrm{C}^{18} \mathrm{O}\right)}=\frac{1-\exp \left(-\tau_{13}\right)}{1-\exp \left(-\tau_{18}\right)}=\frac{1-\exp \left(-\lambda \tau_{18}\right)}{1-\exp \left(-\tau_{18}\right)} .
$$



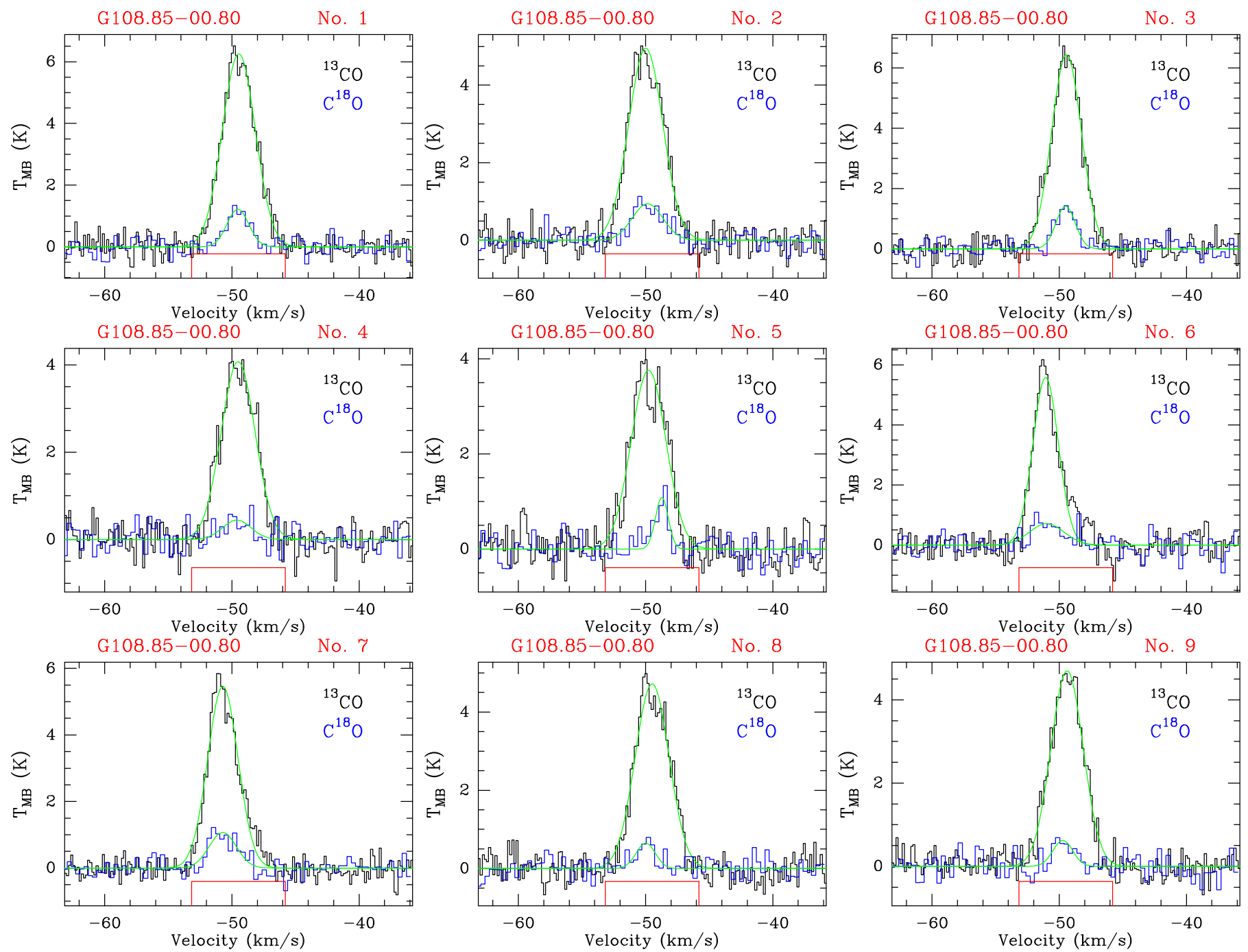

Figure 8. The ${ }^{13} \mathrm{CO}$ (black line) and $\mathrm{C}^{18} \mathrm{O}$ (blue line) lines within each extracted $850 \mu \mathrm{m}$ core (see Figure 7). The green lines show the Gaussian fits in each spectrum. The red window indicates the velocity range of the corresponding ${ }^{13} \mathrm{CO}$ and $\mathrm{C}^{18} \mathrm{O}$ integrated-intensity maps (see Figure 2).

(The complete figure set (117 images) is available.)

Equation (1) assumes a single excitation temperature for both molecules and throughout the line of sight and assumes $\tau_{13}=$ $\lambda \tau_{18}$, where $\lambda$ is the abundance ratio between ${ }^{13} \mathrm{C}^{16} \mathrm{O}$ and ${ }^{12} \mathrm{C}^{18} \mathrm{O}$. Here $\lambda$ can be derived from the relation in Wilson \& Rood (1994) and Pineda et al. (2013) to be

$$
\lambda=\frac{\left[{ }^{13} \mathrm{C}^{16} \mathrm{O}\right]}{\left[{ }^{12} \mathrm{C}^{18} \mathrm{O}\right]}=\frac{58.8 R_{\mathrm{GC}}+37.1}{4.7 R_{\mathrm{GC}}+25.05},
$$

where $R_{\mathrm{GC}}$ is the galactocentric distance. We only consider the sources detected with main-beam brightness temperatures ${ }^{1{ }^{13} \mathrm{CO}}>3 \sigma$ and $T_{\mathrm{C}^{18} \mathrm{O}}>3 \sigma$ (see Table 3). Furthermore, the excitation temperature $T_{\mathrm{ex}}$ is derived from the radiative transfer equation

$$
\begin{gathered}
J(T)=\mathrm{T}_{0} /\left[\exp \left(\mathrm{T}_{0} / T\right)-1\right], \\
T_{\mathrm{MB}}=f\left[J\left(T_{\mathrm{ex}}\right)-J\left(T_{\mathrm{bg}}\right)\right][1-\exp (-\tau)],
\end{gathered}
$$

where $f$ is the beam filling factor that we assume as $f=1$, $T_{\mathrm{bg}}=2.73 \mathrm{~K}$ is the cosmic microwave background temperature, and $T_{0}=h \nu / k=5.29 \mathrm{~K}$ for the $J=1-0$ transition of
${ }^{13} \mathrm{CO}$ (Wong et al. 2008). We then obtain the molecular ${ }^{13} \mathrm{CO}$ column density $N\left({ }^{13} \mathrm{CO}\right)$ from the relation (Bourke et al. 1997)

$$
\begin{aligned}
N\left({ }^{13} \mathrm{CO}\right)_{\text {thin }}= & \frac{T_{\mathrm{ex}}+0.88}{1-\exp \left(-5.29 / T_{\mathrm{ex}}\right)} \\
& \cdot \frac{2.42 \times 10^{14}}{J\left(T_{\mathrm{ex}}\right)-J\left(T_{\mathrm{bg}}\right)} \int T_{\mathrm{MB}}\left({ }^{13} \mathrm{CO}\right) d v,
\end{aligned}
$$

where $N\left({ }^{13} \mathrm{CO}\right)_{\text {thin }}$ and $v$ are in units of $\mathrm{cm}^{-2}$ and $\mathrm{km} \mathrm{s}^{-1}$, respectively. We then apply a correction factor $\tau /(1-\exp (-\tau))$ to the ${ }^{13} \mathrm{CO}$ column density (Pineda et al. 2010; Liu et al. 2013):

$$
N^{\prime}\left({ }^{13} \mathrm{CO}\right)_{\text {corrected }}=N\left({ }^{13} \mathrm{CO}\right)_{\text {thin }} \times \frac{\tau_{13}}{1-\exp \left(-\tau_{13}\right)} .
$$

Finally, the molecular hydrogen column $N_{\mathrm{H}_{2}}$ was calculated, assuming that the $\left[\mathrm{H}_{2} /{ }^{13} \mathrm{CO}\right]$ abundance ratio is $7 \times 10^{5}$ (Frerking et al. 1982).

The ${ }^{13} \mathrm{CO}$ clump mass is given by the integral of the column density across the source via the formula (Kauffmann 
Table 3

Derived Parameters of Extracted CO Clumps

\begin{tabular}{|c|c|c|c|c|c|c|c|c|c|}
\hline Name & $\begin{array}{c}\text { FWHM }^{\mathrm{a}} \\
\operatorname{arcsec}\end{array}$ & $\begin{array}{c}R_{\mathrm{eff}}^{\mathrm{b}} \\
\mathrm{pc}\end{array}$ & $\underset{\mathrm{km} \mathrm{s}^{-1}}{\sigma_{\mathrm{C}^{18}}}$ & $\begin{array}{c}N_{\mathrm{H}_{2}}^{13} \mathrm{CO} \\
10^{21} \mathrm{~cm}^{-2}\end{array}$ & $\begin{array}{c}n_{\mathrm{H}_{2}}^{13} \mathrm{CO} \\
10^{3} \mathrm{~cm}^{-3}\end{array}$ & $\begin{array}{c}\sum^{13} \mathrm{CO} \\
\mathrm{g} \mathrm{cm}^{-2}\end{array}$ & $\begin{array}{c}M_{\mathrm{H}_{2}}^{13} \mathrm{CO} \\
M_{\odot}\end{array}$ & $\begin{array}{c}M_{\mathrm{vir}} \\
M_{\odot}\end{array}$ & $\alpha_{\text {vir }}$ \\
\hline G098.50-03.24_1 & 162.0 & 0.75 & $0.39 \pm 0.08$ & $7.7 \pm 2.0$ & $4.2 \pm 1.1$ & $0.089 \pm 0.023$ & $240.8 \pm 61.4$ & $87.8 \pm 17.5$ & $0.36 \pm 0.12$ \\
\hline G098.50-03.24_2 & 128.4 & 0.59 & $0.25 \pm 0.05$ & $7.0 \pm 1.3$ & $4.0 \pm 0.8$ & $0.068 \pm 0.013$ & $114.4 \pm 21.7$ & $44.5 \pm 9.1$ & $0.39 \pm 0.11$ \\
\hline G108.85-00.80_1 & 166.3 & 1.55 & $0.85 \pm 0.08$ & $41.5 \pm 7.0$ & $10.9 \pm 1.8$ & $0.488 \pm 0.082$ & $5630.1 \pm 945.5$ & $392.1 \pm 36.8$ & $0.07 \pm 0.01$ \\
\hline G108.85-00.80_2 & 106.8 & 1.00 & $0.64 \pm 0.06$ & $68.3 \pm 12.6$ & $27.5 \pm 5.1$ & $0.788 \pm 0.145$ & $3749.7 \pm 690.7$ & $190.6 \pm 18.8$ & $0.05 \pm 0.01$ \\
\hline G108.85-00.80_3 & 164.5 & 1.53 & $1.04 \pm 0.13$ & $25.5 \pm 7.0$ & $4.9 \pm 1.4$ & $0.217 \pm 0.060$ & $2440.1 \pm 671.5$ & $474.2 \pm 59.7$ & $0.19 \pm 0.06$ \\
\hline
\end{tabular}

Notes.

${ }^{\mathrm{a}}$ The extracted clump sizes with the Gaussclumps procedure (see Section 3.2).

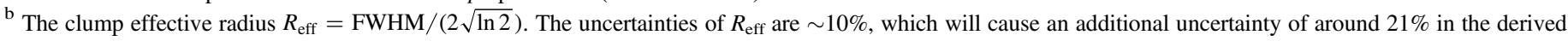
masses.

(This table is available in its entirety in machine-readable form.)

et al. 2008)

$$
M_{\mathrm{H}_{2}}^{13} \mathrm{CO}=\mu_{\mathrm{H}_{2}} m_{\mathrm{H}} D^{2} \int N_{\mathrm{H}_{2}} d \Omega,
$$

where $\mu_{\mathrm{H}_{2}}=2.8, m_{\mathrm{H}}=1.008 \mathrm{u}, D$, and $\Omega$ are the mean molecular weight, mass of a hydrogen atom, distance, and solid angle of the source, respectively. The masses of all extracted ${ }^{13} \mathrm{CO}$ clumps are listed in Table 3.

\subsection{Virial Analysis}

The virial theorem can be used to test whether fragments are in a stable state. Under the assumption of a simple spherical fragment with a density distribution of $\rho=r^{-2}$, ignoring magnetic fields and bulk motions of the gas, the virial mass of a fragment can be estimated from the formula (MacLaren et al. 1988; Evans 1999)

$$
M_{\mathrm{vir}} \simeq 126 R_{\mathrm{eff}} \Delta V_{\mathrm{C}^{18} \mathrm{O}}^{2}\left(M_{\odot}\right),
$$

where $R_{\text {eff }}=$ FWHM $/(2 \sqrt{\ln 2})$ is the effective radius of the fragment in pc, and $\Delta V_{\mathrm{C}^{8} \mathrm{O}}$ (listed in Tables 2 and 4) is the FWHM of the line profile in $\mathrm{km} \mathrm{s}^{-1}$. Here $\Delta V_{\mathrm{C}^{18} \mathrm{O}}$ is the measured $\mathrm{C}^{18} \mathrm{O}$ line width using Gaussian line fitting. For a typical cold cloud $(<20 \mathrm{~K})$, the thermal width is only a few tenths narrower than the observed line width; thus, the observed line width is presumed to be representative of the turbulent velocity structure. The spatial resolution of the $\mathrm{C}^{18} \mathrm{O}$ data is somewhat larger than the sizes of individual cores; hence, we just consider the $\mathrm{C}^{18} \mathrm{O}$ spectrum within 1 pixel corresponding to the peak position of each core (see error analysis in Section 4.1). The virial parameter $\alpha_{\text {vir }}$ is defined by $\alpha_{\mathrm{vir}}=M_{\mathrm{vir}} / M$. The virial masses and virial parameters are listed in Tables 3 and 5.

\subsection{Dust Mass and Surface Density}

We assume that the dust emission is optically thin and the gas-to-dust ratio is 100 . The fragment masses are calculated using dust opacity $\kappa_{\nu}=0.0182 \mathrm{~cm}^{2} \mathrm{~g}^{-1}$ at $850 \mu \mathrm{m}$ (Kauffmann et al. 2008), assuming a gas-to-dust mass ratio of 100 for a model of dust grains with thin ice mantles at a gas density of $10^{6} \mathrm{~cm}^{-3}$ (Ossenkopf \& Henning 1994). The total mass, $M_{\mathrm{H}_{2}}^{850 \mu \mathrm{m}}$, of the $850 \mu \mathrm{m}$ sources can therefore be calculated via the formula (Kauffmann et al. 2008)

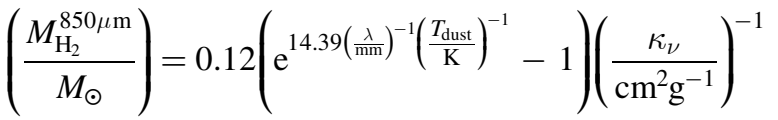

$$
\begin{aligned}
& \times\left(\frac{S_{\nu}}{\mathrm{Jy}}\right)\left(\frac{D}{\mathrm{kpc}}\right)^{2}\left(\frac{\lambda}{\mathrm{mm}}\right)^{3},
\end{aligned}
$$

where $\lambda$ is the observed wavelength in $\mathrm{mm}, T_{\text {dust }}$ is the dust temperature in $K, S_{\nu}$ is the integrated flux in Jy, and $D$ is the distance to the source in kpc. For all fragments, we adopt the associated excitation temperature of ${ }^{13} \mathrm{CO} J=1-0$ as an approximate dust temperature (Liu et al. 2013). The surface density $(\Sigma)$ can be derived from $\Sigma=M /\left(\pi R_{\text {eff }}^{2}\right)$ in units of $\mathrm{g} \mathrm{cm}^{-2}$, where $R_{\text {eff }}$ is the effective radius of the fragment, and FWHM is the source size. These corresponding parameters are also listed in Tables 4 and 5 .

\section{Discussion}

We have surveyed 64 PGCCs with CO and in the $850 \mu \mathrm{m}$ continuum in the second quadrant of the Milky Way. The CO observations have low spatial resolution and therefore trace relatively extended molecular clouds and clumps at larger scales, while the $850 \mu \mathrm{m}$ continuum observations have relatively high spatial resolution and are used to trace the dense cores at smaller scales, embedded within the molecular clouds. Investigating the fragments at different scales and comparing their differences will help us to improve our understanding of the early stages of the star formation process. By combining $\mathrm{CO}$ isotopologues $\left({ }^{13} \mathrm{CO}\right.$ and $\left.\mathrm{C}^{18} \mathrm{O}\right)$, important physical parameters can be quantitatively estimated to characterize and increase our knowledge of the clump properties. Five of the PGCCs, G108.85-00.80, G112.52+08.38, $\mathrm{G} 120.67+02.66$, G120.98+02.66, and G121.92-01.71, are distributed over larger regions than the scan map size $\left(14^{\prime} \times 14^{\prime}\right)$, and further observations over a larger region are needed for a complete analysis.

\subsection{Error Analysis of Different Beam Sizes}

The $\mathrm{CO}$ and $850 \mu \mathrm{m}$ observations have beam sizes of around $52^{\prime \prime}$ and $14^{\prime \prime}$, respectively. To derive some parameters of $850 \mu \mathrm{m}$ cores, such as velocity dispersion and temperature, we have to use the $\mathrm{CO}$ line data observations for estimation. However, there is no simple way of assigning $\mathrm{CO}$ emission to 
Table 4

Observed Parameters of Extracted $850 \mu \mathrm{m}$ Cores

\begin{tabular}{|c|c|c|c|c|c|c|c|c|c|}
\hline Name & $\begin{array}{l}\text { Offset (R.A. Decl.) })^{\mathrm{a}} \\
\qquad\left({ }^{\prime \prime \prime}\right)\end{array}$ & $\begin{array}{l}V_{13} \mathrm{CO}_{1} \\
\mathrm{~km} \mathrm{~s}^{-1}\end{array}$ & $\begin{array}{l}\Delta V^{13} \mathrm{CO}^{-1} \\
\mathrm{~km} \mathrm{~s}^{-1}\end{array}$ & $\begin{array}{c}T 13 \mathrm{CO} \\
\mathrm{K}\end{array}$ & $\begin{array}{l}V_{\mathrm{C}^{18}} \mathrm{O} \\
\mathrm{km}^{-1}\end{array}$ & $\begin{array}{l}\Delta V_{\mathrm{C}^{18} \mathrm{O}} \\
\mathrm{km} \mathrm{s}^{-1}\end{array}$ & $\underset{\mathrm{K}}{T_{\mathrm{C}^{18} \mathrm{O}}}$ & $\tau{ }^{13} \mathrm{CO}$ & $\begin{array}{c}T_{\mathrm{ex}}\left({ }_{\mathrm{K}}^{13} \mathrm{CO}\right) \\
\end{array}$ \\
\hline G108.85-00.80_1 & $78.42,89.09$ & $-49.46 \pm 0.02$ & $3.06 \pm 0.05$ & $6.26 \pm 0.29$ & $-49.53 \pm 0.10$ & $2.01 \pm 0.23$ & $1.22 \pm 0.23$ & $1.36 \pm 0.57$ & $17.12 \pm 1.95$ \\
\hline G108.85-00.80_2 & $-49.32,-80.21$ & $-49.99 \pm 0.03$ & $3.17 \pm 0.07$ & $4.95 \pm 0.32$ & $-49.84 \pm 0.16$ & $2.84 \pm 0.34$ & $0.94 \pm 0.22$ & $1.29 \pm 0.70$ & $14.25 \pm 2.19$ \\
\hline G108.85-00.80_3 & $64.49,46.21$ & $-49.43 \pm 0.02$ & $2.76 \pm 0.05$ & $6.44 \pm 0.30$ & $-49.50 \pm 0.08$ & $1.79 \pm 0.18$ & $1.41 \pm 0.21$ & $1.72 \pm 0.51$ & $16.68 \pm 1.22$ \\
\hline G108.85-00.80_4 & $107.96,184.14$ & $-49.51 \pm 0.03$ & $3.14 \pm 0.08$ & $4.07 \pm 0.29$ & $\cdots$ & $\ldots$ & $\ldots$ & $\ldots$ & $\cdots$ \\
\hline G108.85-00.80_5 & $-49.38,-113.27$ & $-49.76 \pm 0.04$ & $3.22 \pm 0.09$ & $3.76 \pm 0.32$ & $-48.72 \pm 0.13$ & $1.19 \pm 0.33$ & $1.08 \pm 0.26$ & $2.71 \pm 1.03$ & $9.96 \pm 0.92$ \\
\hline
\end{tabular}

Note.

The absolute coordinate of each source is listed in Table 1.

(This table is available in its entirety in machine-readable form.) 
Table 5

Derived Parameters of Extracted $850 \mu \mathrm{m}$ Cores

\begin{tabular}{|c|c|c|c|c|c|c|c|c|c|c|}
\hline Name & $\begin{array}{c}\mathrm{FWHM}^{\mathrm{a}} \\
\operatorname{arcsec}\end{array}$ & $\begin{array}{c}R_{\mathrm{eff}}^{\mathrm{b}} \\
\mathrm{pc}\end{array}$ & $\underset{\mathrm{km} \mathrm{s}^{-1}}{\sigma_{\mathrm{C}^{18} \mathrm{O}}}$ & $\begin{array}{c}S_{850} \\
\text { Jy }\end{array}$ & $\begin{array}{c}N_{\mathrm{H}_{2}}^{850 \mu \mathrm{m}} \\
10^{22^{2}} \mathrm{~cm}^{-2}\end{array}$ & $\begin{array}{c}n_{\mathrm{H}_{2}}^{850 \mu \mathrm{m}} \\
10^{4} \mathrm{~cm}^{-3}\end{array}$ & $\begin{array}{l}\sum^{850 \mu \mathrm{m}} \\
\mathrm{g} \mathrm{cm}^{-2}\end{array}$ & $\begin{array}{c}M_{\mathrm{H}_{2}}^{850 \mu \mathrm{m}} \\
M_{\odot}\end{array}$ & $\alpha_{\text {vir }}$ & $\begin{array}{l}\text { Infrared }^{\mathrm{c}} \\
W I S E\end{array}$ \\
\hline G108.85-00.80_1 & 30.2 & 0.28 & $0.85 \pm 0.10$ & 2.19 & $6.4 \pm 1.5$ & $5.0 \pm 0.9$ & $0.403 \pm 0.072$ & $154.0 \pm 27.6$ & $0.46 \pm 0.10$ & Yes \\
\hline G108.85-00.80_2 & 26.5 & 0.25 & $1.21 \pm 0.14$ & 1.43 & $4.6 \pm 1.4$ & $6.5 \pm 1.7$ & $0.464 \pm 0.122$ & $135.9 \pm 35.7$ & $0.65 \pm 0.19$ & No \\
\hline G108.85-00.80_3 & 30.7 & 0.29 & $0.76 \pm 0.08$ & 1.55 & $7.0 \pm 1.4$ & $3.5 \pm 0.4$ & $0.289 \pm 0.034$ & $113.8 \pm 13.3$ & $0.57 \pm 0.09$ & No \\
\hline G108.85-00.80_4 & 18.7 & 0.17 & $\ldots$ & 0.53 & $18.8 \pm 0.8$ & $12.4 \pm 1.6$ & $0.621 \pm 0.081$ & $90.5 \pm 11.8$ & $\ldots$ & No \\
\hline G108.85-00.80_5 & 16.3 & 0.15 & $0.51 \pm 0.14$ & 0.37 & $5.3 \pm 1.7$ & $14.1 \pm 2.7$ & $0.615 \pm 0.118$ & $68.2 \pm 13.1$ & $0.33 \pm 0.11$ & No \\
\hline
\end{tabular}

Notes.

${ }^{\mathrm{a}}$ The extracted clump sizes with the Gaussclumps procedure (see Section 3.2).

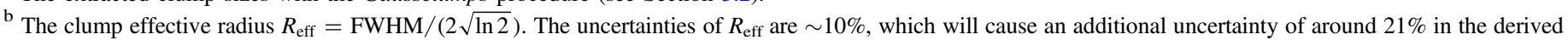
masses.

${ }^{c}$ The point-source cross-identification using the AllWISE Data in the VizieR Online Data Catalog (see Section 2.3).

(This table is available in its entirety in machine-readable form.)

each $850 \mu \mathrm{m}$ core when they form a tight cluster, for example, in $\mathrm{G} 172.85+02.27$. That is, the $\mathrm{CO}$ emission toward each one of these six $850 \mu \mathrm{m}$ cores is contaminated by emission from nearby cores. If the $850 \mu \mathrm{m}$ cores are not located in a tight cluster, it seems that we can use a reasonable filling factor, $f=(14 / 52)^{2}$, to estimate the excitation temperature assuming all integrated intensity of the ${ }^{13} \mathrm{CO}$ clump emits from the dense and isolated $850 \mu \mathrm{m}$ core. However, the large $52^{\prime \prime}$ beam means that the velocity gradients from, e.g., accretion along filaments, rotation, and even molecular outflows, will overestimate the velocity dispersion of each $850 \mu \mathrm{m}$ core. Therefore, this will lead to high uncertainties for our estimation. The velocity dispersion, virial mass, and virial parameter of the $850 \mu \mathrm{m}$ cores will be overestimated, and the excitation temperature at the position of each $850 \mu \mathrm{m}$ core will be underestimated.

\subsection{Infrared Emission}

The extended $12 \mu \mathrm{m}$ emission originates mainly from polycyclic aromatic hydrocarbons (Watson et al. 2008), which are excited by UV radiation at the interface between the expanding $\mathrm{H}$ II region and the ambient interstellar medium (Zhang et al. 2016a). The extended $22 \mu \mathrm{m}$ emission is mostly produced by relatively hot dust (Anderson et al. 2012; Faimali et al. 2012) and is a good tracer of early star formation activity.

Figures 4 and 5 compare the infrared emission of WISE 12 and $22 \mu \mathrm{m}$ with the ${ }^{13} \mathrm{CO}$ emission contours. The morphological distribution of the ${ }^{13} \mathrm{CO}$ emission is correlated or uncorrelated with the 12 and $22 \mu \mathrm{m}$ emission, which corresponds to infrared-bright and infrared-dark PGCCs, respectively. We find that $\sim 30 \%$ of PGCCs are infrared-bright after visually inspecting the image, while $\sim 70 \%$ sources are infrared-dark. We also note that $\sim 15 \%$ of the infrared-dark PGCCs have more than one infrared-bright core, which is also correlated with a peak in the ${ }^{13} \mathrm{CO}$ emission. Positionally matching the AllWISE catalog (Cutri et al. 2013, 2014) with the extracted $850 \mu \mathrm{m}$ sources (see Table 5), we find that 74 of the $117850 \mu \mathrm{m}$ cores have corresponding WISE infrared point sources. Those with or without infrared point sources may be protostellar or infrared quiet/starless cores, respectively (Yuan et al. 2017). This suggests that the infrared-dark PGCCs with infrared-bright cores are more evolved but younger than the infrared-bright PGCCs.

We find no infrared dust bubbles (Churchwell et al. 2006, 2007), which would show strong $22 \mu \mathrm{m}$ emission surrounded by a ringlike $12 \mu \mathrm{m}$ emission shell. The 12 and $22 \mu \mathrm{m}$ emissions have no significant morphological differences. Compact H II regions or bright infrared cores may have an effect on the evolution of early star formation (Zhang et al. 2017b). The integrated-intensity maps of some PGCCs show steep gradients, e.g., G098.50-03.24, G127.88+02.66, and G151.08+04.46 (see Figure 2). This suggests that the molecular clouds have been compressed by nearby warm clouds, such as G035.39-00.33 (Liu et al. 2018b), possibly indicating cloud-cloud collisions, such as in the case of G178.28-00.61 (T. Zhang et al. 2018, in preparation).

\subsection{Fragmentation}

In Figure 3, we present the ${ }^{13} \mathrm{CO}$ and $\mathrm{C}^{18} \mathrm{O}$ line spectra extracted from the identified $468{ }^{13} \mathrm{CO}$ clumps in our sample of 64 PGCCs. They typically have sizes of $\sim 0.2-2 \mathrm{pc}$. This shows that each of the PGCCs fragments into an average of $\sim 7.3{ }^{13} \mathrm{CO}$ clumps. The G144.84+00.76 PGCC fragments into 14 clumps. We suggest that the fragmentation is ubiquitous and a necessary process of the early stage of star formation. Analysis shows that most of the clumps are associated with $\mathrm{CO}$ structures. Only 68 sources of the ${ }^{13} \mathrm{CO}$ clumps were not detected with $\mathrm{C}^{18} \mathrm{O}$ lines, suggesting that most of the clumps are relatively dense.

Figure 7 shows examples of $850 \mu \mathrm{m}$ emission maps with extracted cores overlaid. In total, 117 cores are extracted at $850 \mu \mathrm{m}$. Less than half (28) of the 64 PGCCs have been detected with $850 \mu \mathrm{m}$ continuum, indicating that each PGCC fragments into 4.2 cores, on average, with an effective radius of $0.03-0.48 \mathrm{pc}$. We suggest that the number of fragments is strongly associated with the fragment size, and the results might also be dependent on the sensitivity (Pokhrel et al. 2018).

\subsection{Mass-size Relation}

Li (2017) derived a scaling relation of $m \sim r^{5 / 3}$ to describe the properties of the gravitationally bound structures, where the multiplication factor of the relationship is determined by the level of ambient turbulence. A higher level of turbulence leads to a higher mass at a given scale. It has been found that the scaling provides a good description of the fragments observed from subparsec scales to those of a few pc (Zhang et al. 2017a). In Figure 9, the red dashed line shows the mass-size relation derived assuming $m \sim r^{5 / 3}$ from Li (2017) and Zhang et al. (2017a). Figure 9 also displays the mass-size relation derived from a linear fit to the data. In general, the results obtained from 


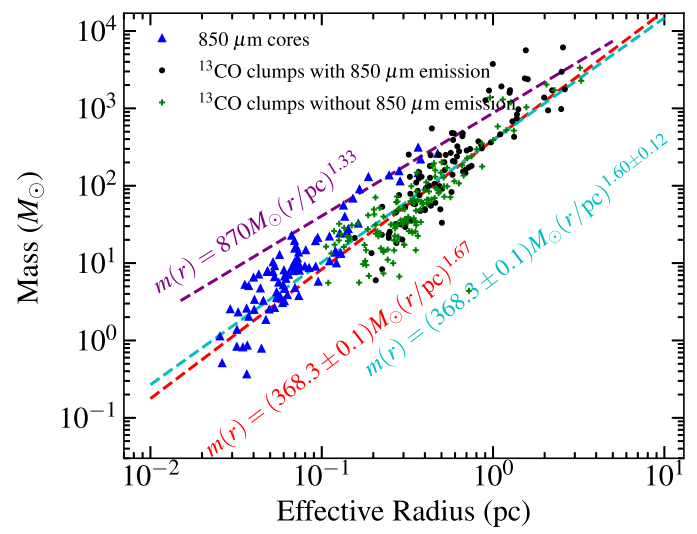

Figure 9. Mass-radius distributions of Gaussian $850 \mu \mathrm{m}$ cores (blue triangles) and ${ }^{13} \mathrm{CO}$ clumps with (black dots) and without (green crosses) $850 \mu \mathrm{m}$ emission. Their masses and effective radii are listed in Tables 3 and 5, respectively. The purple line delineates the threshold introduced by Kauffmann \& Pillai (2010), separating the regimes into high-mass and low-mass starforming candidates. The red line shows a power-law fit using least-squares fitting in log-space with a fixed exponent 1.67 to the mass-size relation for clumps that undergo quasi-isolated gravitational collapse in a turbulent medium (Li 2017; Zhang \& Li 2017). The cyan line presents a power-law fit of all the data points using least-squares fitting. The corresponding formulae are also shown near the lines.

these fits are very similar. This clearly demonstrates that these clumps do not obey "Larson's third law," where the power-law exponent is $\sim 1.9$ (Larson 1981; Solomon et al. 1987) but are consistent with the prediction made in $\mathrm{Li}$ (2017).

Having established the relation, it is possible to use the properties of the observed clumps to estimate the turbulence in the ambient medium. In our sample, we found that our structures satisfy the relationship, $m(r)=(368.3 \pm 0.1) M_{\odot}(r / \mathrm{pc})^{1.67}$ (see Figure 9). The multiplication factor is small compared to that found in high-mass star-forming regions by Urquhart et al. (2014), who had a relationship of $m(r)=2630 M_{\odot}(r / \mathrm{pc})^{1.67}$, and Zhang et al. (2017a), $m(r)=7079 M_{\odot}(r / \mathrm{pc})^{1.67}$. The samples in Zhang et al. (2017a) are more massive and denser than those in Urquhart et al. (2014). At a given scale, the masses of gas condensation in our PGCC sample are around 1/10 of that of typical Galactic high-mass star-forming regions (Urquhart et al. 2014). Using the scaling relation presented in Li (2017), this implies that the energy dissipation rate of the ambient turbulence should be 1/30 of that of the Galactic massive star-forming regions, where we expect the observed velocity dispersion of the molecular gas in our PGCC sample to be $1 / 3$ times the average Galactic value on a given scale. ${ }^{31}$ In general, the level of turbulence in the PGCC sample is significantly lower than the Galactic average. This is consistent with our previous findings in Zhang et al. (2016b).

\subsection{Low-mass Star Formation}

In Figure 9, we present the mass-size plane for the extracted ${ }^{13} \mathrm{CO}$ clumps and $850 \mu \mathrm{m}$ cores. Comparison with the highmass star formation threshold of $m(r)>870 M_{\odot}(r / p c)^{1.33}$ empirically proposed by Kauffmann \& Pillai (2010) allows us to determine whether these fragments are capable of giving

\footnotetext{
31 These numbers are obtained using the scaling relations presented in $\mathrm{Li}$ (2017), where the mass-size relation is determined by $m \approx$ $\epsilon_{\text {cascade }}^{2 / 3} \eta^{-2 / 3} G^{-1} r^{5 / 3}$ (where $m$ is the critical mass, $r$ is the source size, and $\epsilon_{\text {cascade }} \approx \eta \sigma_{\mathrm{v}}^{3} / l$ is the turbulence energy dissipation rate of the ambient medium).
}

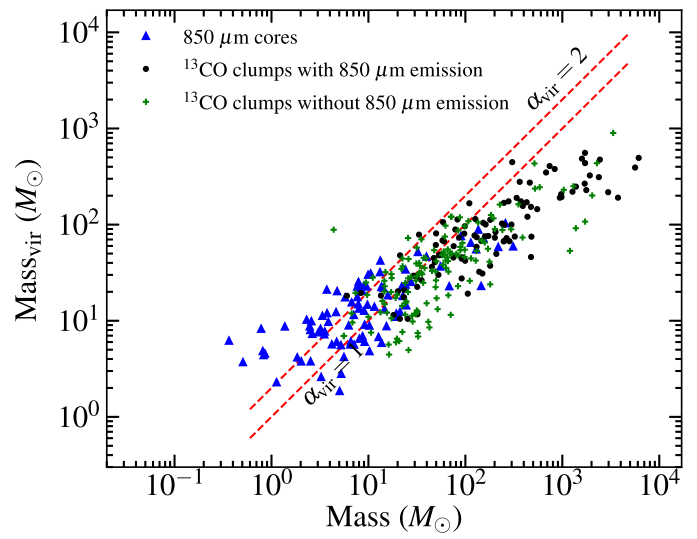

Figure 10. Mass vir - mass distributions of Gaussian $850 \mu \mathrm{m}$ cores (blue triangles) and ${ }^{13} \mathrm{CO}$ clumps with (black dots) and without (green crosses) $850 \mu \mathrm{m}$ emission. The parameters are listed in Tables 3 and 5. Two dashed lines delineate the thresholds of $\alpha_{\text {vir }}=1$ and 2 .

birth to massive stars. The data points are mostly distributed below the threshold, given by the purple dashed line. Therefore, it appears that the majority of ${ }^{13} \mathrm{CO}$ clumps and $850 \mu \mathrm{m}$ cores are low-mass star-forming region candidates.

In Figure 10, we present virial mass versus fragment mass distributions for the ${ }^{13} \mathrm{CO}$ clumps and $850 \mu \mathrm{m}$ cores. Two dashed lines show the thresholds with virial parameters $\alpha_{\text {vir }}=1$ and 2 . We find that $\sim 26 \%$ of the ${ }^{13} \mathrm{CO}$ clumps have $\alpha_{\text {vir }}>1$, and $\sim 5 \%$ have $\alpha_{\text {vir }}>2$, while $\sim 71 \%$ of the $850 \mu \mathrm{m}$ cores have $\alpha_{\text {vir }}>1$, with $\sim 37 \%$ having $\alpha_{\text {vir }}>2$. This indicates that most of the $850 \mu \mathrm{m}$ cores are gravitationally unbound and either stable or expanding (Hindson et al. 2013), relative to the ${ }^{13} \mathrm{CO}$ clumps. It is also likely that the kinetic energy is larger than the gravitational energy, suggesting that such cores have to be confined by some external pressure (Bertoldi \& McKee 1992; Pattle et al. 2015). Therefore, a long timescale for star formation is required for most of our local PGCCs, or they will never form stars.

Mass surface density, $\Sigma$, is a commonly used parameter to assess the high-mass star formation potential. Urquhart et al. (2014) suggested that the surface density of $0.05 \mathrm{~g} \mathrm{~cm}^{-2}$ might represent a minimum threshold of efficient massive star formation, as is suitable for parsec-scale clumps. According to this threshold, parts of the ${ }^{13} \mathrm{CO}$ clumps are potential candidates for massive star formation. However, we note that most of the candidates have a typical size less than $1.0 \mathrm{pc}$. Traficante et al. (2018) argued that $\Sigma=0.12 \mathrm{~g} \mathrm{~cm}^{-2}$ may represent the minimum surface density at clump scales for high-mass star formation to occur, based on the analysis of dynamic activity associated with their parent clump. Krumholz \& McKee (2008) suggested that a minimum mass surface density of $1 \mathrm{~g} \mathrm{~cm}^{-2}$ is required to prevent fragmentation into low-mass cores through radiative feedback, thus allowing highmass star formation. For the ${ }^{13} \mathrm{CO}$ clumps and $850 \mu \mathrm{m}$ cores in this work, we find that the mean values of surface densities are 0.13 and $0.39 \mathrm{~g} \mathrm{~cm}^{-2}$, respectively (see Figure 12). Therefore, the surface densities further prove that some of the ${ }^{13} \mathrm{CO}$ clumps and $850 \mu \mathrm{m}$ cores have the potential to form high-mass stars, but the majority would form low-mass stars.

\subsection{Core Mass Function}

The core mass function (CMF) generally has a comparable slope with the stellar initial mass function and, consequently, 


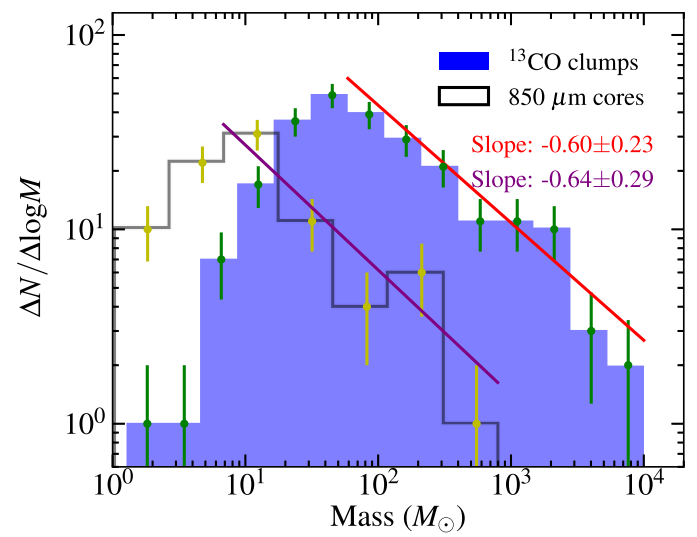

Figure 11. Mass distribution for ${ }^{13} \mathrm{CO}$ clumps (blue) and $850 \mu \mathrm{m}$ cores (black). The parameters are listed in Tables 3 and 5. The slopes of the fitted power-law index are shown in the histogram. The error bars represent the standard deviation of a Poisson distribution $\sqrt{\Delta N / \Delta \log M}$.

the Salpeter power law with a logarithmic slope of -1.35 (Salpeter 1955; Zinnecker \& Yorke 2007). Previous observations show that massive stars usually form in dense clusters, so competitive accretion of protostars from their common gas reservoir was used to explain the observed Salpeter stellar mass distribution for massive stars (Bonnell et al. 2001; Klessen \& Burkert 2001). To investigate the intermediate- and high-mass star formation, in Figure 11, we simply fit the mass spectra in the mass ranges between 400 and $8000 M_{\odot}$ for ${ }^{13} \mathrm{CO}$ clumps and between 7 and $800 M_{\odot}$ for $850 \mu \mathrm{m}$ cores with a linear least-squares method using the obtained clump and core masses in our observations, respectively. The lower mass limit used to define the power-law tail is derived from the peak positions (at $\sim 400 M_{\odot}$ for the clumps and $\sim 7 M_{\odot}$ for the cores) from the low-mass to high-mass end of the mass spectrum distributions in Figure 11. The derived two slopes of the mass spectrum are similar to each other with a clump scope $k_{\text {clump }}=-0.60 \pm$ 0.23 and core slope $k_{\text {core }}=-0.64 \pm 0.29$, which are much flatter than the Salpeter stellar initial mass function and the CMFs of massive star-forming candidates (e.g., Beuther \& Schilke 2004; Bontemps et al. 2010; Ohashi et al. 2016; Csengeri et al. 2017b). For low-mass star-forming objects, Marsh et al. (2016) obtained a slope of $-0.55 \pm 0.07$ in the Taurus L1495 cloud, Elia et al. (2013) derived a slope of $-0.7 \pm 0.3$ for the gas clump distribution in the third Galaxy quadrant, and Kim et al. (2004) derived a shallower mass function slope of $-0.59 \pm 0.32$ for their clump sample named CMa OB1 and G220.8-1.7. The three cases above are consistent with our results. The similar slopes may have resulted from their similar initial conditions. We also have to note that the sample distribution at different distances and the contamination from large-scale structure may lead to uncertain slopes (Moore et al. 2007; Reid et al. 2010).

\subsection{Core Formation Efficiency}

The CFE describes the fraction of the clump mass that has converted into denser cores (Elia et al. 2013; Veneziani et al. 2017). Hence, the CFE is defined as

$$
\mathrm{CFE}=\frac{M_{\text {core }}}{M_{\text {core }}+M_{\text {clump }}},
$$

where $M_{\text {core }}$ is the mass of the $850 \mu \mathrm{m}$ cores, and $M_{\text {clump }}$ is the mass of the ${ }^{13} \mathrm{CO}$ clump that hosts those associated $850 \mu \mathrm{m}$ cores. Considering that $M_{\text {clump }}$ is estimated from the extracted Gaussian clumps, the diffuse gas component of the cloud will be missing. Additionally, the clump masses are estimated by ${ }^{13} \mathrm{CO}$, which will be depleted in low temperatures $(<18 \mathrm{~K}$; Pillai et al. 2007, 2011), hence, $M_{\text {clump }}$ will be underestimated. The cores in our sample are considered to be gravitationally bound objects. Using the core and clump masses of the entire sample to estimate the CFE, we get a CFE of $3.0 \% .{ }^{32}$ Of all 64 PGCCs, only $28(43.8 \%)$ are detected at $850 \mu \mathrm{m}$ with emission above $5 \sigma$, indicating a low CFE. Our estimated CFE is much lower than those estimated from the conversion of molecular clouds to clumps across the first quadrant (5\%-8\%; Eden et al. 2012, 2013), the first and second quadrants (5\%-23\%; Battisti \& Heyer 2014), the fourth quadrant (8\%-39\%; Veneziani et al. 2017), and the Galactic center (10\%-13\%; Csengeri et al. 2016).

\subsection{Statistics}

\subsection{1. ${ }^{13}$ CO Clumps and $850 \mu m$ Cores}

Figure 12 presents histograms of the velocity dispersions, optical depths, excitation temperatures, surface densities, and virial parameters for the ${ }^{13} \mathrm{CO}$ clumps and $850 \mu \mathrm{m}$ cores.

The velocity dispersion $\left(\sigma_{\mathrm{v}}\right)$ histogram in Figure 12 shows that the median value is $0.40 \pm 0.15 \mathrm{~km} \mathrm{~s}^{-1}$ for the ${ }^{13} \mathrm{CO}$ clumps, smaller than that $\left(0.57 \pm 0.19 \mathrm{~km} \mathrm{~s}^{-1}\right)$ of the $850 \mu \mathrm{m}$ cores, indicating that the $850 \mu \mathrm{m}$ cores are more dynamically active at a small scale and consistent with the fact that the $850 \mu \mathrm{m}$ cores are mainly located at the peak positions of the ${ }^{13} \mathrm{CO}$ clumps (see Figure 6) or that some cores with IR emission are forming stars. It seems that the $850 \mu \mathrm{m}$ cores are generally more turbulent than the ${ }^{13} \mathrm{CO}$ clumps. Another possibility is that there is active star formation injecting turbulence into the $850 \mu \mathrm{m}$ cores.

From the optical depth $\left(\tau^{13} \mathrm{CO}\right)$ distribution in Figure 12, we find that the median values are $0.89 \pm 0.65$ for the ${ }^{13} \mathrm{CO}$ clumps and $1.75 \pm 0.46$ for the $850 \mu \mathrm{m}$ cores. Most of the ${ }^{13} \mathrm{CO}$ clumps have optical depths $<1.0$. This indicates that most of the ${ }^{13} \mathrm{CO}$ clumps are more optically thin than the $850 \mu \mathrm{m}$ cores.

The excitation temperature $\left(T_{\mathrm{ex}}\right)$ histogram in Figure 12 shows that the median value is $14.1 \pm 5.0 \mathrm{~K}$ for the ${ }^{13} \mathrm{CO}$ clumps and $15.3 \pm 2.6 \mathrm{~K}$ for the $850 \mu \mathrm{m}$ cores. Considering the $850 \mu \mathrm{m}$ cores are smaller than the ${ }^{13} \mathrm{CO}$ beam, the filling factors should be $f<1$. However, we adopt $f=1$ to estimate excitation temperature, which will lead to underestimating the excitation temperature for the $850 \mu \mathrm{m}$ cores (see also the error analysis in Section 4.1). It suggests that the internal parts of the clumps have higher temperatures than the outer parts, probably indicating an internal heating mechanism.

The surface density $(\Sigma)$ histogram in Figure 12 shows that the median value is $0.10 \pm 0.04 \mathrm{~g} \mathrm{~cm}^{-2}$ for the ${ }^{13} \mathrm{CO}$ clumps, while it is $0.33 \pm 0.15 \mathrm{~g} \mathrm{~cm}^{-2}$ for the $850 \mu \mathrm{m}$ cores. The median value of the surface densities of the $850 \mu \mathrm{m}$ cores is much larger than that of the ${ }^{13} \mathrm{CO}$ clumps, indicating that some $850 \mu \mathrm{m}$ cores that are gravitationally bound are denser and represent the precise locations where the stars would form inside the clumps.

\footnotetext{
${ }^{32}$ Here we consider all the extracted clumps and cores.
} 

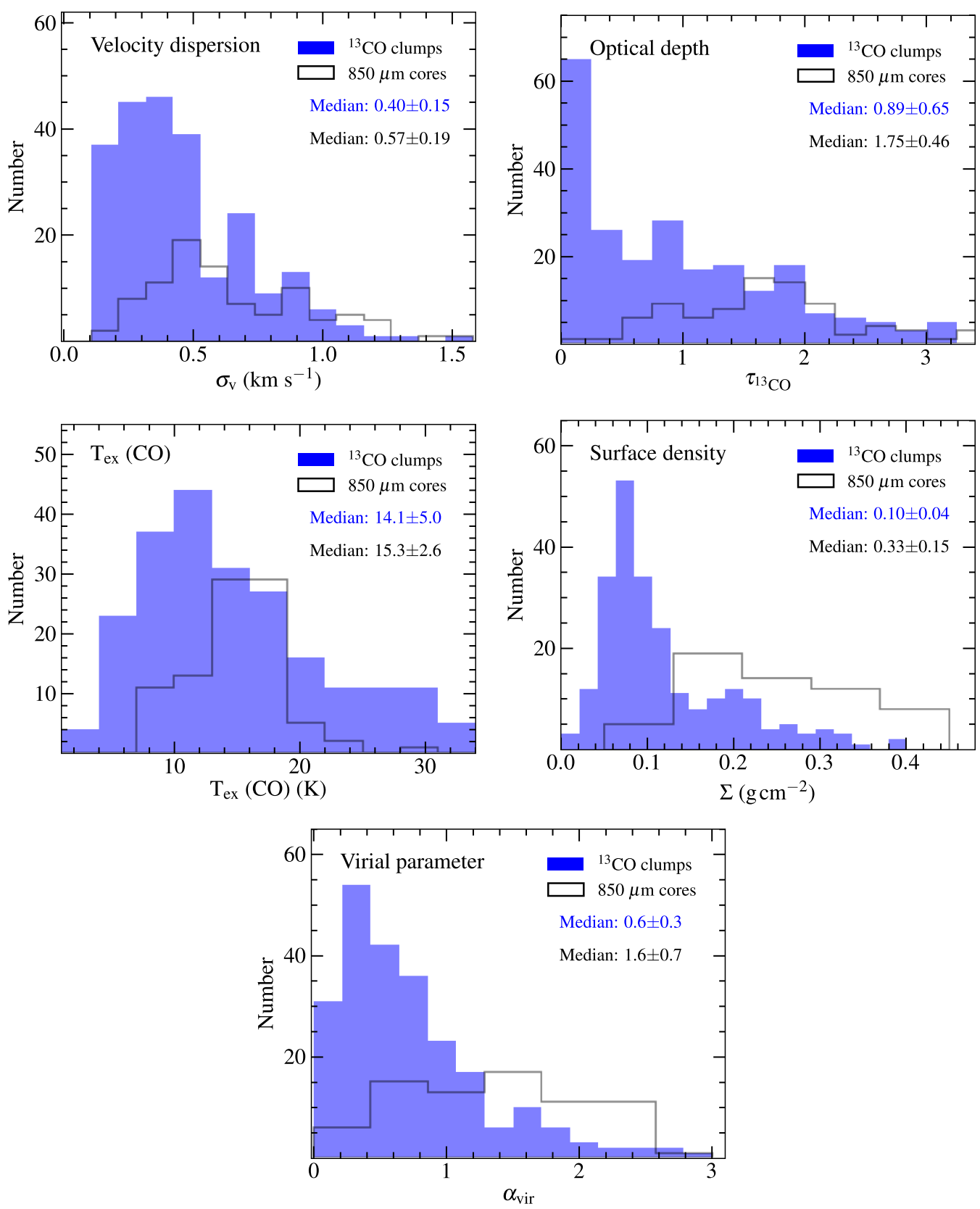

Figure 12. Histograms of velocity dispersion, optical depth, excitation temperature, surface density, and virial parameter for ${ }^{13} \mathrm{CO}$ clumps (blue) and $850 \mu \mathrm{m}$ cores (black). The parameters are listed in Tables 2-5. The corresponding median value is presented in each frame. The uncertainty on each median calculated represents the median absolute deviation.

The virial parameter $\left(\alpha_{\text {vir }}\right)$ histogram in Figure 12 shows that the median value is $0.6 \pm 0.3$ for the ${ }^{13} \mathrm{CO}$ clumps, while it is $1.6 \pm 0.7$ for the $850 \mu \mathrm{m}$ cores. The virial parameters above 2.0 may indicate that the fragments have difficulty forming stars (Kauffmann et al. 2008) without the help of external pressure. Based on the virial parameters in this work, most of the ${ }^{13} \mathrm{CO}$ clumps are candidates to form dense cores. Further checking their embedded cores at $850 \mu \mathrm{m}$, the median value of their virial parameters is around 1.6. Therefore, our cores are mostly gravitationally unbound and may be dispersing at the core scale, or estimates based on ${ }^{13} \mathrm{CO}$ overestimate the in-core turbulence. We also note that 76 out of 117 cores at $850 \mu \mathrm{m}$ have WISE counterparts (see Section 4.2). It is likely that many cores have already formed stars and may be in the process of expansion.

\subsubsection{PGCCs with and without $850 \mu m$ Emission}

Figure 13 presents histograms of the velocity dispersions, optical depths, excitation temperatures, surface densities, and virial parameters for the detected ${ }^{13} \mathrm{CO}$ clumps associated with and without $850 \mu \mathrm{m}$ emission.

The velocity dispersion $\left(\sigma_{\mathrm{v}}\right)$ histogram in Figure 13 shows that for PGCCs with and without $850 \mu \mathrm{m}$ emission, the median values are $0.49 \pm 0.20$ and $0.35 \pm 0.13 \mathrm{~km} \mathrm{~s}^{-1}$, respectively. This indicates that the PGCCs with $850 \mu \mathrm{m}$ emission are more dynamically active and turbulent than those without $850 \mu \mathrm{m}$ emission.

The optical depth $\left(\tau^{13} \mathrm{CO}\right)$ histogram (using ${ }^{13} \mathrm{CO}$ as the tracer) in Figure 13 shows that the median values are the same $(0.89 \pm 0.65)$ for PGCCs with and without $850 \mu \mathrm{m}$ emission. 

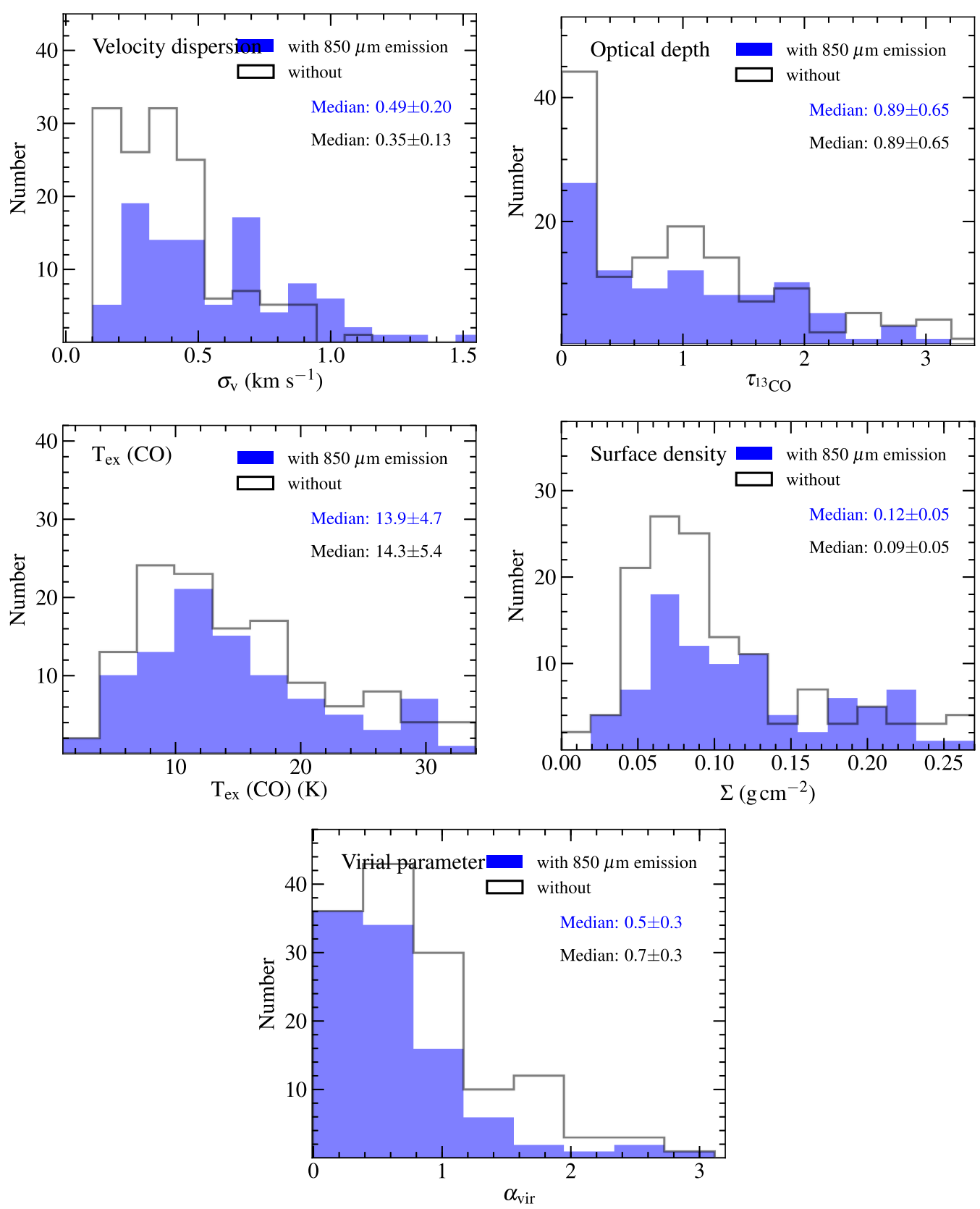

Figure 13. Histograms of velocity dispersion, optical depth, excitation temperature, surface density, and virial parameter for the ${ }^{13} \mathrm{CO}$ clumps in the 64 PGCCs with (blue) and without (black) $850 \mu \mathrm{m}$ extracted emission. The parameters are listed in Tables $2-5$. The corresponding median value is presented in each subhistogram. The uncertainty on each median calculated represents the median absolute deviation.

The excitation temperature $\left(T_{\mathrm{ex}}\right)$ histogram in Figure 13 shows that for PGCCs with and without $850 \mu \mathrm{m}$ emission, the median values are $13.9 \pm 4.7$ and $14.3 \pm 5.4 \mathrm{~K}$, respectively. Therefore, the two groups are practically at the same temperature.

The surface density $(\Sigma)$ histogram in Figure 13 shows that for PGCCs with and without $850 \mu \mathrm{m}$ emission, the median values are $0.12 \pm 0.05$ and $0.09 \pm 0.05 \mathrm{~g} \mathrm{~cm}^{-2}$. This indicates that the densities are similar for both.

The virial parameter $\left(\alpha_{\text {vir }}\right)$ histogram in Figure 13 shows that for PGCCs with and without $850 \mu \mathrm{m}$ emission, the median values are $0.5 \pm 0.3$ and $0.7 \pm 0.3$, respectively. Based on the virial parameters in this work, the PGCCs with $850 \mu \mathrm{m}$ emission probably have a slightly greater potential to form stars than those without $850 \mu \mathrm{m}$ emission.

\subsubsection{Comparison with Other Studies}

Other studies, such as those of IRDCs (e.g., Zhang et al. 2017a), found that the line width of the $\mathrm{C}^{18} \mathrm{O} J=1-0$ line ranges from around 2.0 to $6.0 \mathrm{~km} \mathrm{~s}^{-1}$, the volume density from $870 \mu \mathrm{m}$ continuum measurements is greater than $5.0 \times$ $10^{4} \mathrm{~cm}^{-3}$, and most cores have virial parameters $\alpha_{\mathrm{vir}}<1.0$. Most ATLASGAL clumps and cores (e.g., Csengeri et al. 2014, 2017a; Wienen et al. 2015, 2018; König et al. 2017; Urquhart et al. 2018) are also dynamically active, dense, and gravitationally bound and are high-mass star formation candidates. In this work, however, we find that the 64 PGCCs are dynamically quiescent, optically thin, nondense, and gravitationally unbound, the typical values of which are $\sigma_{\mathrm{v}}<1.5 \mathrm{~km} \mathrm{~s}^{-1}, \tau_{{ }^{13} \mathrm{CO}}<1.0, \Sigma<0.3 \mathrm{~cm}^{-2}$, and $\alpha_{\text {vir }} \gtrsim 1.0$. 
Wu et al. (2012), Liu et al. (2013), and Meng et al. (2013) detected relatively low column densities, velocity dispersions, and high virial parameters $\left(\alpha_{\text {vir }}>1.0\right)$ toward other PGCCs with star formation activity. The consistent results further confirm that the PGCCs are mostly quiescent and lack starforming activity or are most likely at the very initial evolutionary stages of star formation. We note that Zahorecz et al. (2016) found a dozen Planck Galactic cold clumps with the potential to form massive stars and/or star clusters, i.e., they exceed the empirical threshold for massive star formation.

\section{Summary}

To make progress in understanding the early evolution of molecular clouds and dense cores in a wide range of Galactic environments, we carry out an investigation of 64 PGCCs in the second quadrant of the Milky Way using ${ }^{13} \mathrm{CO}, \mathrm{C}^{18} \mathrm{O}$, and $850 \mu \mathrm{m}$ observations. Through the survey, we study their fragmentation and evolution associated with star formation and show a statistical analysis of the extracted ${ }^{13} \mathrm{CO}$ clumps and $850 \mu \mathrm{m}$ cores.

We present the maps of all ${ }^{13} \mathrm{CO}, \mathrm{C}^{18} \mathrm{O}$, and $850 \mu \mathrm{m}$ observations. Using the Gaussclumps procedure in GILDAS, we extracted 468 clumps from the ${ }^{13} \mathrm{CO}$ integrated line intensity maps and 117 cores from the $850 \mu \mathrm{m}$ continuum images. We present all of the observational spectra and derived integrated-intensity maps of ${ }^{13} \mathrm{CO}$ and $\mathrm{C}^{18} \mathrm{O}$ and compute and list the physical parameters of the lines and the extracted fragments.

Using the Bayesian distance calculator (Reid et al. 2016), we derived the distances of all 64 PGCCs in our samples, which are distributed between 0.42 and $5.0 \mathrm{kpc}$ in the second quadrant of the Milky Way. We find that 60 PGCCs are located in the Local and Perseus arms or the associated interarm region, with four PGCCs in the Outer Arm.

Fragmentation analysis shows that each PGCC fragments into 7.3 clumps, on average, in ${ }^{13} \mathrm{CO}$ emission with sizes of around $0.1-3.2 \mathrm{pc}$, and each PGCC detected at $850 \mu \mathrm{m}$ fragments into 4.2 cores at $850 \mu \mathrm{m}$ with effective radii of $0.03-0.48 \mathrm{pc}$. We suggest that the fragmentation number may be associated with the fragment size, and the relationship between fragmentation number and fragment size may reflect the nature of clump and core formation efficiency.

We further studied the properties of the fragments in the mass-size plane. We found that in general, the structure follows a relation that is close to $m \sim r^{1.67}$, which is much shallower than what is predicted by Larson (1981) but consistent if these objects undergo quasi-isolated gravitational collapse in a turbulent medium (Li 2017; Zhang et al. 2017a). At a given scale, the masses of our PGCCs are around 1/10 of that of the typical Galactic massive star-forming regions. This reflects the uniqueness of the PGCC sample: according to ( $\mathrm{Li}$ 2017), the normalization of the mass-size relation is determined by the energy dissipation rate of the ambient turbulence. In our sample, the mass-size relation can be explained if the turbulence observed in these clumps is $1 / 3$ times (measured in velocity dispersion) the average Galactic value.

Statistics indicate that the $850 \mu \mathrm{m}$ cores are more turbulent, more optically thick, and denser than the ${ }^{13} \mathrm{CO}$ clumps, suggesting that most $850 \mu \mathrm{m}$ cores are better star-forming candidates than the ${ }^{13} \mathrm{CO}$ clumps. The excitation temperature histogram may suggest that the inner parts of the clumps have higher temperatures than the outer parts, probably indicating an internal heating mechanism. The PGCCs with $850 \mu \mathrm{m}$ emission are more dynamically active and have more potential to form stars than those without $850 \mu \mathrm{m}$ emission.

Analysis of the clump and core masses, virial parameter, surface density, and mass-size relation suggests that the PGCCs in the second quadrant of the Milky Way have a low CFE of $\sim 3.0 \%$, and most are candidates for low-mass star formation. Comparison with previous studies suggests that the PGCCs are mostly quiescent and lack star-forming activity or are most likely at the very initial evolutionary stages of star formation. As is evident from the physical parameters, it seems clear that the clumps/cores in this PGCC sample are not able to form high-mass stars.

We first thank the anonymous referee for prompting many clarifications of this paper. This work is supported by the National Key Basic Research Program of China (973 Program) 2015CB857100 and the National Natural Science Foundation of China through grants 11703040,11503035 , and 11573036, and the National Key R\&D Program of China 2017YFA0402600 and the CAS International Partnership Program No. 114A11KYSB20160008. C.-P. Zhang acknowledges support by the China Scholarship Council in Germany as a postdoctoral researcher (No. 201704910137). Tie Liu is supported by an EACOA fellowship. C.W.L. was supported by the Basic Science Research Program though the National Research Foundation of Korea (NRF) funded by the Ministry of Education, Science, and Technology (NRF-2016R1A2B4012593). M.J. acknowledges the support of Academy of Finland grant No. 285769. G.-C. Li is supported by the DFG Cluster of Excellence "Origin and Structure of the Universe." K.W. is supported by grant WA3628-1/1 of the German Research Foundation (DFG) through priority program 1573 ("Physics of the Interstellar Medium"). L.V. Toth acknowledges the support of OTKA grant NN-111016. P.S. was financially supported by Grant-in-Aid for Scientific Research (KAKENHI Number 18H01259) of Japan Society for the Promotion of Science (JSPS). We are grateful to the staff at the Qinghai Station of PMO for their assistance during the observations. This publication makes use of data products from the Wide-field Infrared Survey Explorer, which is a joint project of the University of California, Los Angeles, and the Jet Propulsion Laboratory/California Institute of Technology, funded by the National Aeronautics and Space Administration. The JCMT is operated by the East Asian Observatory on behalf of the National Astronomical Observatory of Japan, the Academia Sinica Institute of Astronomy and Astrophysics, the Korea Astronomy and Space Science Institute, the National Astronomical Observatories of China, and the Chinese Academy of Sciences (grant No. XDB09000000), with additional funding support from the Science and Technology Facilities Council of the United Kingdom and participating universities in the United Kingdom and Canada. The SCUBA-2 data mainly taken in this paper were observed under project code M16AL002.

Facilities: PMO, JCMT, WISE.

\section{ORCID iDs}

Chuan-Peng Zhang (i) https://orcid.org/0000-0002-4428-3183 Tie Liu (1) https://orcid.org/0000-0002-5286-2564

Jinghua Yuan (1) https://orcid.org/0000-0001-8060-3538

Patricio Sanhueza (i) https://orcid.org/0000-0002-7125-7685

Di Li @i https://orcid.org/0000-0003-3010-7661 
Ken'ichi Tatematsu (1) https://orcid.org/0000-0002-8149-8546

Ke Wang (1) https://orcid.org/0000-0002-7237-3856

Anthony Marston (10 https://orcid.org/0000-0001-5788-5258

Pak Shing Li (1) https://orcid.org/0000-0001-8077-7095

Patrick M. Koch (1) https://orcid.org/0000-0003-2777-5861

Yuefang Wu $\odot$ https://orcid.org/0000-0002-5076-7520

Tianwei Zhang (i) https://orcid.org/0000-0002-1466-3484

Paul F. Goldsmith (i) https://orcid.org/0000-0002-6622-8396

L. V. Tóth (1) https://orcid.org/0000-0002-5310-4212

Kee-Tae Kim (10) https://orcid.org/0000-0003-2412-7092

\section{References}

Anderson, L. D., Zavagno, A., Deharveng, L., et al. 2012, A\&A, 542, A10 Battisti, A. J., \& Heyer, M. H. 2014, ApJ, 780, 173

Belloche, A., Schuller, F., Parise, B., et al. 2011, A\&A, 527, A145

Bergin, E. A., \& Tafalla, M. 2007, ARA\&A, 45, 339

Bertoldi, F., \& McKee, C. F. 1992, ApJ, 395, 140

Beuther, H., \& Schilke, P. 2004, Sci, 303, 1167

Bintley, D., Holland, W. S., MacIntosh, M. J., et al. 2014, Proc. SPIE, 9153 , 915303

Bonnell, I. A., Bate, M. R., Clarke, C. J., \& Pringle, J. E. 2001, MNRAS, 323, 785

Bontemps, S., Motte, F., Csengeri, T., \& Schneider, N. 2010, A\&A, 524, A18 Bourke, T. L., Garay, G., Lehtinen, K. K., et al. 1997, ApJ, 476, 781 Buckle, J. V., Drabek-Maunder, E., Greaves, J., et al. 2015, MNRAS, 449, 2472 Chapin, E. L., Berry, D. S., Gibb, A. G., et al. 2013, MNRAS, 430, 2545 Churchwell, E., Povich, M. S., Allen, D., et al. 2006, ApJ, 649, 759 Churchwell, E., Watson, D. F., Povich, M. S., et al. 2007, ApJ, 670, 428 Csengeri, T., Bontemps, S., Wyrowski, F., et al. 2017a, A\&A, 601, A60 Csengeri, T., Bontemps, S., Wyrowski, F., et al. 2017b, A\&A, 600, L10 Csengeri, T., Urquhart, J. S., Schuller, F., et al. 2014, A\&A, 565, A75 Csengeri, T., Weiss, A., Wyrowski, F., et al. 2016, A\&A, 585, A104

Cutri, R. M., Wright, E. L., Conrow, T., et al. 2013, yCat, 2328

Cutri, R. M., Wright, E. L., Conrow, T., et al. 2014, yCat, 2328

Dame, T. M., Hartmann, D., \& Thaddeus, P. 2001, ApJ, 547, 792

Dame, T. M., Ungerechts, H., Cohen, R. S., et al. 1987, ApJ, 322, 706

Dempsey, J. T., Friberg, P., Jenness, T., et al. 2013, MNRAS, 430, 2534

Drabek, E., Hatchell, J., Friberg, P., et al. 2012, MNRAS, 426, 23

Eden, D. J., Moore, T. J. T., Morgan, L. K., Thompson, M. A., \& Urquhart, J. S. 2013, MNRAS, 431, 1587

Eden, D. J., Moore, T. J. T., Plume, R., \& Morgan, L. K. 2012, MNRAS, 422, 3178

Elia, D., Molinari, S., Fukui, Y., et al. 2013, ApJ, 772, 45

Evans, N. J., II 1999, ARA\&A, 37, 311

Faimali, A., Thompson, M. A., Hindson, L., et al. 2012, MNRAS, 426, 402

Frerking, M. A., Langer, W. D., \& Wilson, R. W. 1982, ApJ, 262, 590

Gómez, L., Wyrowski, F., Schuller, F., Menten, K. M., \& Ballesteros-Paredes, J. 2014, A\&A, 561, A148

Heyer, M. H., \& Terebey, S. 1998, ApJ, 502, 265

Hindson, L., Thompson, M. A., Urquhart, J. S., et al. 2013, MNRAS, 435, 2003

Holland, W. S., Bintley, D., Chapin, E. L., et al. 2013, MNRAS, 430, 2513

Johnstone, D., Boonman, A. M. S., \& van Dishoeck, E. F. 2003, A\&A, 412,157

Johnstone, D., di Francesco, J., \& Kirk, H. 2004, ApJL, 611, L45

Juvela, M., He, J., Pattle, K., et al. 2018, A\&A, 612, A71

Juvela, M., Ristorcelli, I., Montier, L. A., et al. 2010, A\&A, 518, L93

Kauffmann, J., Bertoldi, F., Bourke, T. L., Evans, N. J., II, \& Lee, C. W. 2008, A\&A, 487, 993

Kauffmann, J., \& Pillai, T. 2010, ApJL, 723, L7

Kim, B. G., Kawamura, A., Yonekura, Y., \& Fukui, Y. 2004, PASJ, 56, 313

Kim, J., Lee, J.-E., Liu, T., et al. 2017, ApJS, 231, 9

Klessen, R. S., \& Burkert, A. 2001, ApJ, 549, 386

König, C., Urquhart, J. S., Csengeri, T., et al. 2017, A\&A, 599, A139

Könyves, V., André, P., Men'shchikov, A., et al. 2015, A\&A, 584, A91

Kramer, C., Stutzki, J., Rohrig, R., \& Corneliussen, U. 1998, A\&A, 329, 249

Krumholz, M. R., \& McKee, C. F. 2008, Natur, 451, 1082
Lane, J., Kirk, H., Johnstone, D., et al. 2016, ApJ, 833, 44 Larson, R. B. 1981, MNRAS, 194, 809

Li, G.-X. 2017, MNRAS, 465, 667

Liu, T., Kim, K.-T., Juvela, M., et al. 2018a, ApJS, 234, 28

Liu, T., Li, P. S., Juvela, M., et al. 2018b, ApJ, 859, 151

Liu, T., Wu, Y., Mardones, D., et al. 2015, PKAS, 30, 79

Liu, T., Wu, Y., \& Zhang, H. 2012, ApJS, 202, 4

Liu, T., Wu, Y., \& Zhang, H. 2013, ApJL, 775, L2

Liu, T., Zhang, Q., Kim, K.-T., et al. 2016, ApJS, 222, 7

MacLaren, I., Richardson, K. M., \& Wolfendale, A. W. 1988, ApJ, 333, 821

Marsh, K. A., Kirk, J. M., André, P., et al. 2016, MNRAS, 459, 342

Meng, F., Wu, Y., \& Liu, T. 2013, ApJS, 209, 37

Moore, T. J. T., Bretherton, D. E., Fujiyoshi, T., et al. 2007, MNRAS, 379, 663

Moore, T. J. T., Plume, R., Thompson, M. A., et al. 2015, MNRAS, 453, 4264

Motte, F., Bontemps, S., \& Louvet, F. 2017, arXiv:1706.00118

Myers, P. C., Linke, R. A., \& Benson, P. J. 1983, ApJ, 264, 517

Nutter, D., \& Ward-Thompson, D. 2007, MNRAS, 374, 1413

Ohashi, S., Sanhueza, P., Chen, H.-R. V., et al. 2016, ApJ, 833, 209

Ossenkopf, V., \& Henning, T. 1994, A\&A, 291, 943

Parsons, H., Dempsey, J. T., Thomas, H. S., et al. 2018, ApJS, 234, 22

Pattle, K., Ward-Thompson, D., Kirk, J. M., et al. 2015, MNRAS, 450, 1094

Pillai, T., Kauffmann, J., Wyrowski, F., et al. 2011, A\&A, 530, A118

Pillai, T., Wyrowski, F., Hatchell, J., Gibb, A. G., \& Thompson, M. A. 2007, A\&A, 467, 207

Pineda, J. L., Goldsmith, P. F., Chapman, N., et al. 2010, ApJ, 721, 686

Pineda, J. L., Langer, W. D., Velusamy, T., \& Goldsmith, P. F. 2013, A\&A, 554, A103

Planck Collaboration, Ade, P. A. R., Aghanim, N., et al. 2011a, A\&A, 536, A1 Planck Collaboration, Ade, P. A. R., Aghanim, N., et al. 2011b, A\&A, 536, A7

Planck Collaboration, Ade, P. A. R., Aghanim, N., et al. 2011c, A\&A, 536, A22

Planck Collaboration, Ade, P. A. R., Aghanim, N., et al. 2011d, A\&A, 536, A23

Planck Collaboration, Ade, P. A. R., Aghanim, N., et al. 2016, A\&A, 594, A28

Pokhrel, R., Myers, P. C., Dunham, M. M., et al. 2018, ApJ, 853, 5

Rathborne, J. M., Jackson, J. M., \& Simon, R. 2006, ApJ, 641, 389

Reid, M. A., Wadsley, J., Petitclerc, N., \& Sills, A. 2010, ApJ, 719, 561

Reid, M. J., Dame, T. M., Menten, K. M., \& Brunthaler, A. 2016, ApJ, 823, 77

Rumble, D., Hatchell, J., Gutermuth, R. A., et al. 2015, MNRAS, 448, 1551

Sanhueza, P., Jackson, J. M., Foster, J. B., et al. 2012, ApJ, 756, 60

Salpeter, E. E. 1955, ApJ, 121, 161

Sofue, Y. 2011, PASJ, 63, 813

Solomon, P. M., Rivolo, A. R., Barrett, J., \& Yahil, A. 1987, ApJ, 319, 730

Stutzki, J., \& Guesten, R. 1990, ApJ, 356, 513

Tang, M., Liu, T., Qin, S.-L., et al. 2018, ApJ, 856, 141

Tatematsu, K., Liu, T., Ohashi, S., et al. 2017, ApJS, 228, 12

Traficante, A., Fuller, G. A., Smith, R. J., et al. 2018, MNRAS, 473, 4975

Urquhart, J. S., König, C., Giannetti, A., et al. 2018, MNRAS, 473, 1059

Urquhart, J. S., Moore, T. J. T., Csengeri, T., et al. 2014, MNRAS, 443, 1555

Veneziani, M., Schisano, E., Elia, D., et al. 2017, A\&A, 599, A7

Wang, K., Zhang, Q., Testi, L., et al. 2014, MNRAS, 439, 3275

Wang, K., Zhang, Q., Wu, Y., \& Zhang, H. 2011, ApJ, 735, 64

Watson, C., Povich, M. S., Churchwell, E. B., et al. 2008, ApJ, 681, 1341

Wienen, M., Wyrowski, F., Menten, K. M., et al. 2015, A\&A, 579, A91

Wienen, M., Wyrowski, F., Menten, K. M., et al. 2018, A\&A, 609, A125

Wilson, T. L., \& Rood, R. 1994, ARA\&A, 32, 191

Wong, T., Ladd, E. F., Brisbin, D., et al. 2008, MNRAS, 386, 1069

Wright, E. L., Eisenhardt, P. R. M., Mainzer, A. K., et al. 2010, AJ, 140, 1868

Wu, Y., Liu, T., Meng, F., et al. 2012, ApJ, 756, 76

Yi, H.-W., Lee, J.-E., Liu, T., et al. 2018, arXiv:1805.05738

Yuan, J., Wu, Y., Ellingsen, S. P., et al. 2017, ApJS, 231, 11

Yuan, J., Wu, Y., Liu, T., et al. 2016, ApJ, 820, 37

Zahorecz, S., Jimenez-Serra, I., Wang, K., et al. 2016, A\&A, 591, A105

Zhang, C.-P., \& Li, G.-X. 2017, MNRAS, 469, 2286

Zhang, C.-P., Li, G.-X., Wyrowski, F., et al. 2016a, A\&A, 585, A117

Zhang, C. P., \& Wang, J. J. 2012, A\&A, 544, A11

Zhang, C.-P., Yuan, J.-H., Li, G.-X., Zhou, J.-J., \& Wang, J.-J. 2017a, A\&A, 598, A76

Zhang, C.-P., Yuan, J.-H., Xu, J.-L., et al. 2017b, RAA, 17, 057

Zhang, T., Wu, Y., Liu, T., \& Meng, F. 2016b, ApJS, 224, 43

Zinnecker, H., \& Yorke, H. W. 2007, ARA\&A, 45, 481 